\title{
Long-Term Portfolio Choice Given Uncertain Personal Savings
}

\author{
Franke, Guenter; Peterson, Sandra; Stapleton, Richard C.
}

Document Version

Final published version

Publication date:

2003

License

CC BY-NC-ND

Citation for published version (APA):

Franke, G., Peterson, S., \& Stapleton, R. C. (2003). Long-Term Portfolio Choice Given Uncertain Personal Savings. LEFIC. Center for Law, Economics and Financial Institutions. LEFIC Working Paper No. 19

Link to publication in CBS Research Portal

\section{General rights}

Copyright and moral rights for the publications made accessible in the public portal are retained by the authors and/or other copyright owners and it is a condition of accessing publications that users recognise and abide by the legal requirements associated with these rights.

\section{Take down policy}

If you believe that this document breaches copyright please contact us (research.lib@cbs.dk) providing details, and we will remove access to the work immediately and investigate your claim. 


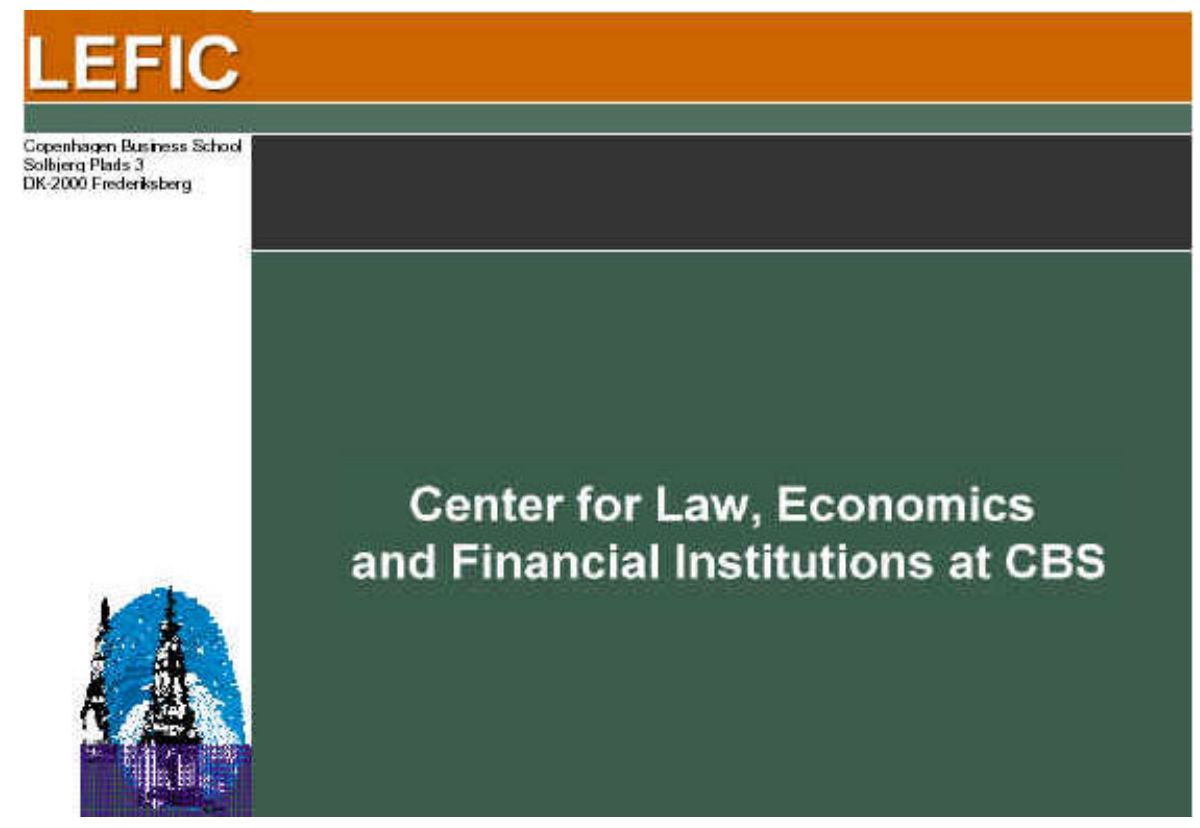

LEFIC WORKING PAPER 2002-19

LONG-TERM PORTFOLIO CHOICE GIVEN UNCERTAIN PERSONAL SAVINGS

Guenter Franke, Sandra Peterson and Richard C. Stapleton

www.cbs.dk/LEFIC 


\title{
Long-Term Portfolio Choice Given Uncertain Personal Savings
}

\author{
Guenter Franke $^{1}$, Sandra Peterson and Richard C. Stapleton ${ }^{2}$
}

January 21, 2003

\footnotetext{
${ }^{1}$ University of Konstanz

${ }^{2}$ University of Strathclyde and University of Melbourne. Previous versions of this paper have been presented at
} 


\section{Abstract \\ Long-Term Portfolio Choice Given Uncertain Personal Savings}

Investors choosing a portfolio strategy, in order to secure a pension at a future date for example, are faced with many uncertainties. One major uncertainty is the amount by which their pension fund will be supplemented by personal savings from a variety of sources such as life insurance contracts, bequests, or property sales. Over long periods of time these uncertainties are likely to be large and difficult to hedge, and hence may have a significant effect on the dynamic portfolio strategy. Drawing on the results of previous literature on the reaction of investors to non-unhedgeable background risk, and on the theory of stochastic dynamic programming, this article derives optimal strategies for investors maximising the expected utility of terminal wealth, where this wealth consists of the value of a pension fund plus accumulated personal savings. Numerical results, assuming that the market portfolio and the expectation of personal savings follow (possibly) correlated geometric Brownian motions, are derived to illustrate the effects of the size and uncertainty of the personal savings, as well as the effect of the resolution of the uncertainty in them over time. The computation uses a new technique for implementing the stochastic dynamic programming. This involves a binomial approximation, in two dimensions, which ensures that the computations are feasible for relatively long-term problems. 


\section{Introduction}

We consider the investment strategy of an individual who invests over a period of time for a lump sum to be received at a given terminal date $T$. The individual has investible wealth at time 0 equal to $W_{0}$ and chooses a dynamic investment strategy over the periods $1,2, \ldots, T$. The individual buys state-contingent claims on the market portfolio and a shortterm riskless bond. At time $T$, the investor either consumes the proceeds of the investment or turns them into a pension. In addition to the proceeds of the investment fund, $W_{T}$, the individual's wealth will be supplemented at $T$ by personal savings from a variety of sources such as bequests, life insurance contracts, property sales, labor income or sales of shares in private businesses. We denote these accumulated personal savings as $S_{T}$. Hence the total wealth at time $T$ is $W_{T}+S_{T}$. The individual is assumed to maximise the expected value of a utility function $u\left(W_{T}+S_{T}\right)$. The personal savings $S_{T}$ are assumed to be exogenous, risky and non-hedgeable. The savings are non-hedgeable due to the personal nature of the risks. If the individual takes out insurance contracts against personal risks, the savings $S_{T}$ are defined net of the cash flows arising from these insurance contracts.

In the above setup we abstract from personal consumption-savings decisions. These are taken as exogenous and they partly contribute to the assumed stochastic process for $S_{T}$. There are hence two stochastic processes in the model. These are the process followed by the value of the market portfolio and the, possibly correlated, process for the expectation of personal savings, $E_{t}\left(S_{T}\right)$, where $t \leq T$. While the process of the market portfolio has positive drift, the savings process has no drift since it is an information process. In this model the personal savings can only be accessed in the future at time $T$. However we assume that the individual is able to borrow against the expected savings. For example, if the individual expects to receive a bequest which will contribute to personal savings at time $T$, he may choose to borrow money at time $t$ in order to invest in stocks. Summarizing, the individual's problem is to choose a dynamic investment strategy for his investible wealth in the presence of an unhedgeable savings risk which evolves over time according to a given stochastic process.

The paper is organized as follows. In section 2 our problem will be related to the literature. Section 3 of this paper sets up the maximisation problem faced by the investor. It highlights the essential difference between a model where the investor has risky personal savings, $S_{T}$ and one where he does not. It also explains our assumptions regarding the information process for $S_{T}$. Section 4 derives the conditions for optimal portfolio policies and characterises these policies. Section 5 describes the computational procedure and section 6 illustrates the optimal portfolio strategies using various numerical examples. 


\section{Portfolio Models, Labor-Income Risk and Asset Alloca- tion}

The possible effect of personal savings out of labor income on portfolio choice has been reported in a number of recent studies. These are well summarised in Campbell and Viceira (2002). It is well known that an agent facing a non-tradable background risk becomes more risk averse to a second, additive tradable risk, given reasonable restrictions on utility. Also, in a single period model, Franke, Stapleton and Subrahmanyam (1998) (FSS) show that agents with different levels of background risk may demand options as well as simple stockbond portfolios. Campbell and Viceira discuss two effects of uncertain labor income risk. First, the existence of other income prospects tends to substitute for bonds in the investor's portfolio. ${ }^{1}$ Hence a relatively young investor with extensive future earnings prospects will tend to have a higher proportion of stocks than an investor at a later stage of his working life. However, this effect is reduced if the income prospects are uncertain. In line with the literature on background risk, in effect the investor becomes more risk averse to the market risks and, hence, buys less stocks.

Viceira (2001) optimises the intertemporal investment-consumption policy of an investor who has uncertain labor income. In his model, labor income follows a geometric process and any savings out of labor income are invested in the portfolio. The single risky asset also follows a possibly correlated geometric process. He finds that the ratio of portfolio wealth to labor income is stationary. Using a log-linear approximation he derives an optimal portfolio policy which has a constant stock proportion. He finds that when labor income risk is independent of the asset return risk, employed investors hold a larger fraction of their savings in the risky asset than retired investors. Koo (1998) and Heaton and Lucas (1997) also derive optimal consumption and portfolio policies with stochastic labor income. Koo uses a continuous time model and shows that the optimal level of risk taking is lower in the presence of an uninsurable labor income risk. Heaton and Lucas, in an infinite horizon model, do not find any significant effect of labor income risk on portfolio composition.

In our model there is a strict separation between the traded investment account and the personal savings account. This is obvious if the savings arise only at the final date as may be typical for bequests, the payoffs from life insurance contracts or from property sales

\footnotetext{
${ }^{1}$ FSS themselves build on the previous analysis of the impact of background risk on portfolio choice. See for example Kimball (1990)(1993), Gollier and Pratt (1996), Nachman (1982), Kihlstrom, Romer and Williams (1981) and Pratt and Zeckhauser (1987). A good summary of the effect of personal-savings risks on portfolio strategies can be found in Gollier (2001). One paper that applies these concepts to the consumptionportfolio problem is Elmendorf and Kimball (2000). In a two-date model they show that a standard risk averse investor takes less tradable risk in the presence of labor income risk.
} 
such as equity stakes in companies or real estate. Even if savings accumulate over time, in our setup money cannot be added to the investment account, and cannot be withdrawn from the account until the terminal date. In practice, many pension investment accounts are separated from personal savings accounts due to taxation regulations. Pension accounts may have tax concessions, but these are given at the expense of some flexibility. The money has to remain in the account until a fixed retirement date. In the meantime personal circumstances change, and this changes the optimal asset allocation in the investment account. Our model allows us to derive an optimal dynamic strategy for the investment account, in the light of changing personal circumstances.

Similar to Viceira's model, the ratio of tradable wealth to non-tradable expected personal savings is the key determinant of the investment policy. However, in our finite horizon model, this ratio varies as the return on the risky asset and the expectation of the personal savings evolve over time. In contrast to Viceira, we find a significant impact of personal savings risk on optimal portfolio allocations, even when the market risk and the personal savings risks are uncorrelated. The effect of the background risk is to make investors more conservative (hold more bonds) as well as to reduce variation over time in the stock-bond ratio as traded wealth changes. In addition we find that established results in the literature that an independent background risk affects the convexity of the investors' demand, extend to a multi-period economy. ${ }^{2}$

Secondly in our model we can vary the speed of resolution of uncertainty regarding the outcome of personal savings. If uncertainty about the level of $S_{T}$ is resolved early in the period between $t$ and $T$, portfolio strategies will differ considerably from the case of late resolution of uncertainty. For example, the case where an uncertain legacy is revealed only at time $T$ differs considerably from the case where information is gained gradually over time regarding the size of savings out of labor income. Finally, in our model, the effects of introducing borrowing constraints can be analysed in a multi-period setting.

As pointed out in Campbell and Viceira (2002), the multi-period portfolio problem in the presence of risky personal savings does not allow for neat analytical solutions. Like a num-

\footnotetext{
${ }^{2}$ There are a number of reasons why the portfolios reported in this paper appear to differ from those reported in Viceira (2001). First, since in Viceira, labor income arises over time and savings are added to the portfolio, the stock proportions do not mean the same thing in the two models. In Viceira the proportions relate to an increasing wealth over time as savings accumulate. Second, in Viceira's infinite horizon model the wealth to income ratio is stationary, so changes due for example to market returns have only a transitory effect. Third, Viceira uses a log-linear approximation, which, together with his assumption of constant relative risk aversion, has the effect of negating any direct effects of an independent background risk on the optimal portfolio policy. There is only an indirect effect through the consumption to wealth elasticity. It is precisely these direct effects that are the main focus of this paper. See Appendix section 8.4 for a review of Viceira's log-linear approximation.
} 
ber of previous authors, we test various conjectures by running numerical simulations where optimal policies are derived using stochastic dynamic programming. ${ }^{3}$ We assume, in line with many previous authors, that investors have constant relative risk averse (CRRA) utility functions. ${ }^{4}$. We also assume that the market portfolio and the information process of the personal savings follow lognormal processes, which can be approximated by joint logbinomial processes. In order to analyse realistic problems where the volatility of personal savings changes over time we employ the recombining lattice technique introduced by Nelson and Ramaswamy (1990) and extended to multivariate processes by Ho, Stapleton and Subrahmanyam (1995) (HSS). This allows us to reduce the number of nodes in the bivariate lattice which makes the dynamic programming feasible for realistic problems.

The results in this paper should be seen as complementary to recent contributions on portfolio strategies in the absence of labor income risks. These include Kandel and Stambaugh (1996), Brennan, Schwartz and Lagnado (1997), Brandt (1999), Barberis (2000) and Lynch (2001) who study the effects of predictability of asset returns on portfolio strategies. Xia (2001) includes learning effects. Balduzzi and Lynch (1999) also discuss the impact of transactions costs. Brennan and Xia (2002) derive the portfolio strategy in the presence of inflation risk.

\section{The Model}

We consider the multi-period portfolio choice of an agent endowed with initial investible wealth, $W_{0}$. The wealth is invested period-by-period so as to maximise the expected utility of terminal wealth at date $T$. However, in contrast to the usual case dealt with in the literature, the maximisation problem is complicated by the existence of uncertain and unhedgeable personal savings, which accumulate through time and will be available at time $T$. These personal savings, $S_{T}$, when added to the terminal value of the portfolio investments, means that total wealth at the terminal date is $W_{T}+S_{T}$. The agent invests $W_{t}$, at each date $t<T$, given that the expectation and the volatility of the personal savings varies over time, as uncertainty is resolved and savings are accumulated. In this dynamic problem, the agent chooses state contingent claims, and solves the problem:

$$
\max E_{t}\left[u\left(W_{T}+S_{T}\right)\right]
$$

\footnotetext{
${ }^{3}$ Other recent work on these problems has used Monte Carlo simulation. See, for example, Detemple, Garcia and Rindisbacher (2000). Alternative methodologies are discussed in Cox and Huang (1989).

${ }^{4}$ This places our results in a long tradition of models starting with Merton (1969), and more recently with Brandt (1999), and others.
} 
subject to a budget constraint, at each date $t=0,1, \ldots, T$, where $u($.$) is the agent's utility$ function for terminal wealth at date $T$.

There are two uncertainties in the model. These are represented by a set of traded states and a set of states which reflect expectations of the future personal savings. The traded states correspond to different outcomes of the market portfolio. We assume that the capital market is perfect and complete with respect to these traded states. In other words, the investor can buy claims on each of the traded states. However, we assume that the agent is not able to buy claims on the different states of his personal savings. The risks associated with the personal savings are non-hedgeable background risks. The decision problem is illustrated in Figure 1. At time $t-1$, the investor buys claims contingent on traded states at time $t$. At each future time $t$ the conditional expected value of the personal savings is revealed and also the the market return. At date $t$ the investor again chooses a portfolio strategy, given the personal-savings expectation and the remaining personal-savings uncertainty at that date and state. The investor chooses his investment strategy, given the state-contingent wealth, $W_{t}$, which was chosen at the previous date, and given the prices of state-contingent claims. We let $\phi_{t-1, t, i}$ be the price per unit probability, at time $t-1$, of a claim paying one unit in state $i$ at time $t$. In general $\phi_{t, t+1}$ is the pricing kernel for claims on $W_{t+1}$, purchased at date $t$. Hence $\phi_{t, t+1}$ is the probability deflated price of the tradable-state contingent claims. The budget constraint that has to be satisfied at time $t-1$ is therefore

$$
E_{t-1}\left(W_{t} \phi_{t-1, t}\right)=W_{t-1}
$$

If there is no personal-savings uncertainty, this portfolio allocation problem has a straightforward solution. Since utility is for wealth at time $T$ only, the investor can solve a single period problem, purchasing a set of state contingent-claims on the traded states at time $T$. This choice can then be interpreted, in a binomial tree model, in terms of a stock-bond portfolio evolving over time. Alternatively, we can think of the problem as a dynamic programming optimisation, whereby the investor chooses, at time $t$, a portfolio of claims on states at time $t+1$, which maximises the indirect utility of those claims, given the optimal reinvestment of those claims over the period $t$ to $T$.

If there exists personal-savings uncertainty, but that uncertainty is wholly resolved at the horizon date $T$ and not before, the above single-period solution can be extended, using the methodology of Franke, Stapleton and Subrahmanyam (1998) (FSS). FSS solve for the optimal set of $W_{T}$ claims, given a zero-mean, independent background risk. This methodology, which involves the derived utility of marketable wealth, given the background risk, may be extendible to the case of a positive-mean and correlated personal-savings risk. The single-period approach is not appropriate in general, however, when the uncertainty of the 
personal-savings is resolved over time. In this case, the portfolio strategy at an intermediate date $t$, depends upon the conditional expectation and volatility of the personal savings. The dynamic programming approach is useful in this case. Starting at date $T-1$, the investor maximises expected utility, subject to the budget constraint, in each possible combination of market-return and personal-savings states. Moving to time $T-2$, the investor chooses a strategy for investing in claims on $T$-1-states, given the subsequent optimal reinvestment strategy that will be chosen at time $T-1$, and so on.

\section{Optimal Portfolio Conditions}

In this section we derive first order conditions for the portfolio optimisation problem and present some analytical results on the characteristics of optimal portfolios in the presence of personal-savings uncertainty.

\subsection{The First Order Conditions}

Since the expectation of personal savings evolves as a stochastic process and since the personal-savings risk is a non-hedgeable background risk, it is not possible to solve the optimal portfolio problem by a single optimisation at date 0 . Hence, we solve the problem by using dynamic programming. However, the stochastic dynamic programming optimisation has to account for the effect, on expected utility, of the background risk of the personalsavings risk. First, we derive the First Order Conditions (I.O.C.) for the optimisation over the final period, from date $T-1$ to date $T$, conditional on the tradable wealth, $W_{T-1}$, inherited at date $T-1$. This optimisation depends on $W_{T-1}$, on the probability distribution of the pricing kernel, $\phi_{T-1, T}$, and on the probability distribution of the personal savings, $S_{T}$. The maximisation problem at time $T-1$ is

$$
\max _{\left\{W_{T}\right\}} E_{T-1}\left[u\left(W_{T}+S_{T}\right)\right]
$$

subject to a budget constraint

$$
E_{T-1}\left(W_{T} \phi_{T-1, T}\right)=W_{T-1},
$$

where $E_{T-1}($.$) is the expectation at time T-1$ over the joint distribution of tradable and personal-savings states at date $T$, given the state of the world (both the tradable and 
personal-savings state) at date $T-1$. The investor maximises the expected value of date $T$ utility in equation (1), subject to the budget constraint (2). $E_{T-1}\left(W_{T} \phi_{T-1, T}\right)$ therefore denotes the total cost of all contingent claims purchased at $T-1$. This cost must equal the available tradable wealth at $T-1, W_{T-1}$.

Define $R_{t, t+1} \equiv W_{t+1} / W_{t}$ as the gross rate of return (one plus the rate of return) of the tradable wealth portfolio between dates $t$ and $t+1$. Then the maximisation problem in (1) can be re-written as

$$
\max _{\left\{R_{T-1, T}\right\}} E_{T-1}\left[u\left(W_{T-1} R_{T-1, T}+S_{T}\right)\right]
$$

subject to the budget constraint

$$
E_{T-1}\left(R_{T-1, T} \phi_{T-1, T}\right)=1 .
$$

Let $R_{t, t+1}^{*}$ denote the optimal gross rate of return and $\lambda_{t}$ be the Lagrange multiplier of the budget constraint. Then differentiating (3) yields the I.O.C.:

$$
E_{T-1}\left[u^{\prime}\left(W_{T-1} R_{T-1, T}^{*}+S_{T}\right) \mid \phi_{T-1, T}\right]=\left(\lambda_{T-1} / W_{T-1}\right) \phi_{T-1, T} .
$$

Note that the conditional expectation in (5) is taken over the personal-savings states at time $T$. The difference between (5) and the I.O.C. in the absence of personal-savings uncertainty is that (5) involves the expectation of the marginal utility. This is consistent with the background risk literature and the analysis of, for example, Kimball (1993). We now take the special case of constant relative risk aversion, where the relative risk aversion is $r>0$. In this case

$$
u\left(W_{T}+S_{T}\right)=\frac{1}{1-r}\left(W_{T}+S_{T}\right)^{1-r}
$$

so that

$$
u^{\prime}\left(W_{T}+S_{T}\right)=\left(W_{T}+S_{T}\right)^{-r}
$$

From (5) and (6) it follows that the I.O.C. in this case is

$$
E_{T-1}\left[\left(R_{T-1, T}^{*}+S_{T} / W_{T-1}\right)^{-r} \mid \phi_{T-1, T}\right]=\left(\lambda_{T-1} / W_{T-1}^{(1-r)}\right) \phi_{T-1, T} .
$$

Now, in order to proceed to the time $T-2$ optimisation, define the indirect utility function of wealth at date $T-1$ as

$$
J\left(W_{T-1}\right)=E_{T-1}\left[u\left(W_{T-1} R_{T-1, T}^{*}+S_{T}\right)\right]
$$


From Bellman's Principle of Optimality, we can write the maximisation problem at time $T-2$ as

$$
\max _{\left\{W_{T-1}\right\}} E_{T-2}\left[J\left(W_{T-1}\right)\right]
$$

subject to the budget constraint

$$
E_{T-2}\left[W_{T-1} \phi_{T-2, T-1}\right]=W_{T-2} .
$$

Using the definition $R_{T-2, T-1} \equiv W_{T-1} / W_{T-2}$, the maximisation problem can then be written:

$$
\max _{\left\{R_{T-2, T-1}\right\}} E_{T-2}\left[J\left(W_{T-2} R_{T-2, T-1}\right)\right]
$$

subject to

$$
E_{T-2}\left[R_{T-2, T-1} \phi_{T-2, T-1}\right]=1 .
$$

The I.O.C. for the date $T-2$ maximisation is then given by

$$
E_{T-2}\left[J^{\prime}\left(W_{T-2} R_{T-2, T-1}\right) \mid \phi_{T-2, T-1}\right]=\frac{\lambda_{T-2}}{W_{T-2}} \phi_{T-2, T-1}
$$

where the expectation is taken over the different personal-savings states at $T-1$. From equation (8) above, differentiating we then have

$J^{\prime}\left(W_{T-2} R_{T-2, T-1}\right)=E_{T-1}\left[u^{\prime}\left(W_{T-1} R_{T-1, T}^{*}+S_{T}\right)\left(R_{T-1, T}^{*}+W_{T-1} \frac{\partial R_{T-1, T}^{*}}{\partial\left(W_{T-2} R_{T-2, T-1}\right)}\right)\right]$.

However, given an optimal policy at time $T-1$, it is not possible to raise the expected utility by marginally changing the return function. It follows that

$$
E_{T-1}\left[u^{\prime}\left(W_{T-1} R_{T-1, T}^{*}+S_{T}\right) W_{T-1} \frac{\partial R_{T-1, T}^{*}}{\partial\left(W_{T-2} R_{T-2, T-1}\right)}\right]=0
$$

and

$$
J^{\prime}\left(W_{T-2} R_{T-2, T-1}\right)=E_{T-1}\left[u^{\prime}\left(W_{T-1} R_{T-1, T}^{*}+S_{T}\right) R_{T-1, T}^{*}\right] .
$$

We can now state the I.O.C. condition for date $t$. In general we have the condition:

$$
E_{t}\left[J^{\prime}\left(W_{t} R_{t, t+1}\right) \mid \phi_{t, t+1}\right]=\frac{\lambda_{t}}{W_{t}} \phi_{t, t+1}, t=0, \ldots, T-2
$$


where

$$
J^{\prime}\left(W_{t-1} R_{t-1, t}\right)=J^{\prime}\left(W_{t}\right)=E_{t}\left[J^{\prime}\left(W_{t} R_{t, t+1}^{*}\right)\right], t=0, \ldots, T-2
$$

The condition in equation (11) has to be satisfied for each $t$ in the dynamic programming implementation of the model. Note that the solution for the I.O.C. in this case where the personal savings of the investor constitute a background risk, is a straightforward generalisation of the dynamic programming solution in the absence of a background risk. If personal savings are certain, the analysis is the same, but the I.O.C in (11) involves no expectation over the personal-savings states.

\subsection{Properties of Optimal Portfolios}

In this section we derive some properties of optimal portfolio strategies in the presence of personal-savings risk. In the absence of personal savings, the investor chooses a strategy which is governed by his preferences and by the evolution of investment opportunities in the capital market. It is well known, from the work of Merton (1969) and others, that the investor follows a stationary investment policy, if the returns on securities follow a stationary random walk and if the investor has constant relative risk averse preferences. Under a stationary investment policy, the investor rebalances his portfolio at each date so as to invest a fixed proportion of wealth into a given security. In a simplified setting, where the investor can only choose between a risk-free asset and a single risky asset, he always invests a constant proportion of wealth in the risky asset. In this case the CRRA investor maintains a constant stock-bond proportion as his wealth changes over time.

Now assume that the investor, in addition to his tradable wealth, has personal savings to be realised at $T$ which are known with certainty at $t=0$. As pointed out by Viceira (2001) and discussed extensively in Campbell and Viceira (2001), the personal savings (human wealth) are a substitute for the payoffs on risk-free bonds. Hence, the investor with personal savings reacts by raising his proportionate investment in stocks. The effect of non-stochastic personal savings can also be analysed by changing the power utility function $u\left(W_{T}\right)=(1-r)^{-1} W_{T}^{1-r}$ to the HARA function $u\left(W_{T}\right)=(1-r)^{-1}\left(W_{T}+S_{T}\right)^{1-r}$. From Kim and Omberg (1996) it is well known that this changes the investor's relative risk aversion from a constant level $r$ to $r /\left(1+S_{T} / W_{T}\right)$. Hence if savings are positive, then relative risk aversion is reduced and is increasing in tradable wealth. We summarise this in the following lemma:

Lemma 1 Given an investor has a utility for terminal date wealth of the constant relative risk aversion class, the effect of positive (negative) non-stochastic personal savings is to 
make the investor behave towards investible wealth like an agent who has HARA utility, no personal savings, and lower (higher) and increasing (decreasing) relative risk aversion.

Hence, when the investor chooses between a risk-free and a single risky asset, the effect of positive, non-stochastic personal savings is to increase the stock proportion. However, this effect declines if tradable wealth increases over time. Higher tradable wealth induces the investor to lower the stock proportion.

Now we consider the effect of personal-savings uncertainty. From Kimball (1993), an investor facing a non-unhedgeable additive background risk, becomes more risk averse towards a tradable risk if he is standard risk averse. ${ }^{5}$ Since the constant relative risk averse utility function is standard risk averse, it follows that the investor faced with personal savings that are uncertain will take less tradable risk than would be the case if the savings were certain. Furthermore, in a single period setting, Franke, Stapleton and Subrahmanyam (1998)(FSS) have shown that a HARA investor chooses an optimal payoff function $W_{T}$ which is not only less sloped (reflecting increased risk aversion), but also more convex in the presence of a zero-mean, independent non-unhedgeable background risk. The investor buys portfolio insurance.

These results from the one-period analysis of the effects of background risk will also be valid in our multiperiod setting if the personal-savings uncertainty is resolved only at the terminal date $T$. If utility is for terminal date wealth, and if no uncertainty regarding personal savings is resolved before $T$, then the investor is faced with what is, in effect, a single period problem. Given a complete market for the tradable claims, the investor can buy at date 0 an optimal portfolio of claims on $W_{T}$ and never revise this portfolio. Hence, resolution of personal-savings uncertainty only at date $T$ makes the optimal portfolio strategy collapse into a one-period optimisation problem. Here the results of Kimball (1993) and FSS can be extended to establish:

\section{Proposition 1 [Effect of Savings Risk: Single Period Case]}

If personal savings are uncertain and independent of the risky asset return, and if there is no resolution of the uncertainty of the personal savings before date $T$, then HARA utility with $r>0$ implies that the investor will choose a more conservative strategy (i.e. he will buy more bonds) than in the case where the savings are non-stochastic and have the same expected value at date 0 . Also, the investor has a linear demand function for contingent

\footnotetext{
${ }^{5}$ Necessary and sufficient conditions for standard risk aversion are declining absolute risk aversion and declining positive absolute prudence.
} 
claims in the absence of savings risk, but a convex demand function in the presence of savings risk.

The intuition behind Proposition 1 is as follows. Since the investor has to bear tradable risk and personal-savings risk he reduces his tradable risk in the presence of personalsavings risk. This 'first-order effect' leads to a more conservative strategy. The statement regarding convexity follows by extending the results in Kimball (1993) and FSS to the case of background risks with a positive mean. Since the adverse effects of background risks are felt most strongly when tradable income is low, the investor buys relatively more claims in these low states. This generates the convexity of the demand function. Note that, in this context, demand is a function $R=R\left(\phi^{-1 / r}\right)$. The proposition follows from the proof of the more general case proved in the appendix, section 8.1.

In the general multi-period case, the uncertainty regarding the size of personal savings will be resolved over the time from 0 to $T$. In this case, as noted above, it is not possible to choose an optimal portfolio strategy at time 0 , without the possibility of future revisions to the strategy. Such revisions will depend on the resolution of the personal-savings uncertainty. The optimal portfolio choice at date $t$ depends upon the expected personal savings and the risk of the savings, as of this date. This raises the question as to whether the results regarding risk taking hold in this more general setting. It turns out that this is the case. In the appendix, section 8.1 we show:

\section{Proposition 2 [Effect of Savings Risk: Multiperiod Case]}

Assume that personal savings are uncertain and independent of the risky asset return, and that the risky asset return is identically and independently distributed over time. Then if some uncertainty about the level of savings is resolved at date $T$, an investor with constant relative risk averse utility with $r>0$ chooses a more conservative strategy (i.e. he will buy more bonds) in each period than in the case where the savings are non-stochastic and have the same expected value at date 0 . Also, in each period, the investor has a linear demand function for contingent claims in the absence of savings risk, but a convex demand function in the presence of savings risk.

The intuition behind Proposition 2 is similar to that behind Proposition 1. In a dynamic setting, the impact of non-unhedgeable personal-savings risk is not only to induce a more conservative payoff function in the final period, but also to do so in each of the preceding periods. This is not really surprising. The investor always buys more bonds from day $t=0$ onwards. Suppose, alternatively, that the investor chooses to delay buying portfolio 
insurance until the last period. Then if the market portfolio return has been low in the previous periods, he would be faced with prohibitively high costs of insurance. In effect the investor needs to protect against changes in expectations of personal savings over time and does so by choosing more bonds and convex payoff functions in each period. In the proof of the Proposition (in the appendix), we also show that the investor's demand for contingent claims is linear in the absence of savings risk. ${ }^{6}$

Next, we consider the time horizon effects on the stock-bond portfolio mix, in the presence of personal-savings uncertainty. Basically, there are two opposing time horizon effects on risk taking. First, whenever the risky asset return was high (low) in the preceding periods, the relative importance of personal savings and the risk of the savings is low (high). In the limit, when the tradable wealth is extremely high, then the investor behaves like an investor without savings. On average, tradable wealth tends to rise over time given a positive drift in the market return process. However, this is not true of expected personal savings, since the savings process is an information process, without drift. It follows that on average the ratio of tradable wealth to expected personal savings increases over time. This tends to reduce risk taking, since by Lemma 1, the investor is, in effect, increasing relative risk averse. However, there is another effect of the time horizon. If uncertainty about the size of personal savings is gradually resolved over time, then there is a reduction in the aversion to tradable risk. As time goes by, the investor is inclined therefore to increase tradable risk taking. We summarise these results in the following Proposition which we state without formal proof:

Proposition 3 [Time Horizon Effects]

Assuming that the investor has Constant Relative Risk Averse preferences:

1. As time goes by, any increase in tradable wealth induces the investor to take less tradable risk, since the investor is increasingly relative risk averse.

2. As time goes by, the resolution of the uncertainty of personal savings induces the investor to take more tradable risk, since the investor is standard risk averse.

3. Hence, the net effect of time passage depends upon the increase in the tradable wealth and the resolution of personal-savings uncertainty.

\footnotetext{
${ }^{6} \mathrm{~A}$ further issue is the effect of the speed of personal-savings uncertainty resolution over time on the optimal portfolio policy in the earlier periods. This is a complex issue and will be analysed using numerical simulations.
} 
Finally, we consider how our results could be affected by the introduction of borrowing constraints. Possible borrowing constraints are particularly important in our model, since the investor may wish to borrow large amounts using the expected personal savings as security. In a dynamic model, the investor has to account for the effect of future binding constraints, as well as current constraints. In the numerical simulations which follow we consider the effect of a of borrowing constraint for cases where personal savings are stochastic. However, it is difficult to derive analytical results for this general case. An indication of the possible effects can be appreciated by considering the case of non-stochastic savings. The effect of future constraints on current policy depends upon the level of risk aversion and in particular on whether $r>1$ or $r<1$. We have the following lemma, that is proved in the appendix 8.2:

\section{Lemma 2 [The Effect of Borrowing Constraints]}

Assume $r>1[=1][<1]$ and that personal savings are non-stochastic. Consider the optimal portfolio policy in the absence of a borrowing constraint. Let $d(u)$ denote a state at $t+1$ subsequent to a state at date $t$ such that the optimal allocation in state $d$ and/or a subsequent state would violate the borrowing constraint (in state $u$ or subsequent state would not violate the borrowing constraint). Then introducing the borrowing constraint has the effect that $R_{t, t+1}^{d} / R_{t, t+1}^{u}$ increases [stays the same] [decreases].

An interpretation of the lemma is as follows. Assume that the investor can borrow up to a certain fraction of his tradable wealth. then the borrowing constraint is more likely to be violated in a low market return state. Also, the investor is less relative risk averse in this state. If the constraint becomes binding, future risk taking is reduced. There are two possible reactions to this: either the investor anticipates this and takes more risk in earlier periods, or he buys more claims on the low states, so as to reduce the borrowing gap. It turns out that if $r<1$ he follows the first strategy and if $r>1$ he follows the second strategy. If $r=1$ the investor is myopic and hence ignores the future constraint in determining his current strategy.

\section{The Numerical Solution Methodology}

As noted by Viceira (2001) and Campbell and Viceira (2001), there are few analytical solutions for this type of multiperiod model. To derive optimal portfolio strategies, we implement the model using numerical analysis. In this section, we describe the methodology used in our numerical simulations. 
We derive optimal portfolio strategies over an $n$-period horizon, using a binomial tree approximation. The market portfolio and the investor's expected personal savings are assumed to follow, possibly correlated, lognormal diffusion processes. The bivariate process for the market portfolio and the expected personal savings is then approximated by a bivariatebinomial process which recombines in two dimensions, as stated in Lemma 3 in the appendix. This uses the technique derived in Peterson and Stapleton (2002), using the method originally suggested by Ho, Stapleton and Subrahmanyam (1995). We assume that the logarithm of the market portfolio value follows a possibly mean-reverting process:

$$
d x_{t}=\mu(x, t) d t+\sigma(t) d z_{1, t},
$$

and that the logarithm of the individual's expected personal savings follows the process:

$$
d y_{t}=\mu(y, t) d t+\sigma(t) d z_{2, t},
$$

where $\mu(y, t)=-\frac{\sigma^{2}(t)}{2}$ so that the expected personal-savings process has a zero drift, and $\rho(x, y)$ is the instantaneous correlation of the two processes.

Having built a tree of states for the two variables: the market portfolio return and the personal-savings expectation, we then solve for optimal portfolios using stochastic dynamic programming. This method relies on solving for the first order conditions described above. Initially, we assume that the investor has unrestricted borrowing possibilities. Hence, he can borrow against his expected personal savings and invest an amount greater than his tradable wealth in the risky asset. Later, we show the effect of various borrowing restrictions (see Table 9), where the I.O.C. cannot always be satisfied.

\section{A Simulation of Optimal-Investment Strategies}

\subsection{Assumptions}

In this section we present some findings on the optimal portfolio strategies that result from solving the first order conditions derived above. We make the following additional assumptions:

1. The market portfolio value and the conditional expectation of the investor's personal savings follow (possibly correlated) lognormal processes.

2. The risk-free rate is given and non-stochastic. 
3. The market return is i.i.d.

4. There are no borrowing restrictions. The investor can borrow or lend at the risk-free rate of interest. ${ }^{7}$

We solve the dynamic programming problem using a standard grid approach. First, given a grid of possible values of $W_{T-1}$, we solve for the optimal allocation $W_{T}$. Then we solve the first order condition for the optimal $W_{T-1}$, given $W_{T-2}$, and so on. Given the optimal contingent claim allocation vectors, $W_{t}$, we figure out the stock-portfolio proportions consistent with these allocations. Here we assume that all bonds are single-period, zero-coupon bonds.

The following results show solutions to the five-year optimisation problem $(T=5)$. We assume the following parameter values in all the reported numerical results:

1. The time period is of length one year. The market return is i.i.d. with an expectation of $11 \%$ per year and a volatility of $20 \%$.

2. The one-period risk-free rate is given and non-stochastic. The zero-coupon bond has a price of 0.95 at the beginning of each period.

3. The initial expected value and annual volatility of the personal savings vary across different simulations and are shown as $E_{0}\left(S_{T}\right)$ and $\sigma_{S}$ in the following tables. Unless specified, $\sigma_{S}$ is constant over time indicating equal resolution of uncertainty about personal savings over time.

4. Except for Table 4 and Figure 3c, the market return and the personal savings are assumed to be independent.

5. The coefficient of relative risk aversion $r$ is assumed to be $r=2$, except in Figure 4, where $r$ varies from 2 to 5 . Without personal-savings this implies a constant optimal stock proportion over time of $64 \%$.

6. Initial investible wealth is $W_{0}=100$.

7. Except where expressly stated to the contrary, we assume that the binomial density used in constructing the binomial process is $n=1^{8}$. This implies that there is a

\footnotetext{
${ }^{7}$ However, note that even without any borrowing constraints, it is never optimal for the investor to take a risk of a personal default, since $u^{\prime}\left(W_{T}+S_{T}\right) \rightarrow \infty$ for $W_{T}+S_{T} \rightarrow 0$.

${ }^{8}$ The binomial density is the number of bifurcations per time period. Hence, the number of tradable states at the end of a period is $(n+1)$, given a state at the beginning of the period. The same is true of the number of savings states.
} 
one-to-one relationship between the wealth allocation at a given date $t$ and the stock proportion chosen at $t-1$. This assumption is relaxed in Table 8 , where results for cases with $n=4$ are reported.

\subsection{The Effect of Uncertain Personal Savings on Optimal Asset Alloca- tion}

The results for a series of simulations are presented in Tables 1-10. In these tables we report the optimal portfolio strategy of the investor in terms of a stock proportion chosen in a given state at a point in time. The stock proportion is a proportion of the investible wealth of the investor. Hence, in Table 1, the investor chooses a proportion 1.30 invested in stocks, i.e. he borrows 0.30 , and invests 1.30 in stocks, at time $t=0$. From time $t=1$ onwards, the bond proportion depends on two stochastic variables: the outcome of the market portfolio and the investor's expected personal savings. At time $t=4$, for example, there are 25 states. Since the binomial trees for the market return and the expectation of the personal savings recombine, there are 5 market states and for each of these 5 personal savings states, a total of $5^{2}=25$. In the tables, the first column displays the state $(i, j)$, where $i$ denotes the number of down moves in the market return and $j$ denotes the number of down moves in expected personal savings. Focusing for the moment on the middle state, $i=2$, of the market return at date 4 , the five possible levels of expected personal savings yield stock proportions ranging from 2.06 to 1.06. The higher is the personal savings expectation, the higher naturally is the amount of leverage chosen by the investor. Looking across the various market return states by focusing on the middle personal savings state, $j=2$, we see that the stock proportion varies from 0.98 in the top market state to 2.82 in the bottom market state. This illustrates the increasing proportional risk aversion effect of personal savings [Lemma 1]. In the bottom states the investor borrows aggressively because the relative level of the expected personal savings is larger in these states.

The portfolio strategy changes over time due first, to the resolution of the uncertainty of the personal savings and second, due to the increase in wealth that occurs as the market return drifts upward. The effect of the uncertainty resolution is to increase the investment in stocks, while the wealth effect reduces the stock investment. In this case the net effect is an increase in the stock investment. Comparing the central nodes: where $i=j$, we can observe an increase in leverage from a stock proportion of 1.30 at $t=0$ to 1.33 at $t=2$ and to 1.36 at $t=4$.

The portfolio strategies which result from the model contrast with those reported by Viceira (2001), who found that an investor with risky personal savings and CRRA utility pursues a 
constant, Merton-like, portfolio strategy over time. The reason for our contrasting results is due to the different assumptions made about the personal-savings process. In Viceira (2001), the investor receives labor income in each period and consumes a constant proportion of the income. In Viceira's analysis, the labor income process follows a geometric random walk. As a result, if labor income increases in a particular period, so does the expectation of future labor income. This implies that both the investible wealth and expected labor income rise in that model. In our model a change in the expectation of personal savings has a radical effect on the ratio of expected personal savings to investible wealth. As can be seen from the table, this in turn has a radical effect on the optimal investment proportions.

\subsection{The Effect of the Relative Size of Personal Savings}

For constant relative risk averse investors, the optimal stock proportion depends on the ratio of expected personal savings to investible wealth, not on the absolute levels of these variables. The effect of the relative size of the risky personal savings is illustrated in Figures 2a-2c. Figure 2a plots the stock proportions, in the middle personal savings node for $t=2$ and $t=4$, or the average of the two nodes nearest to the middle for $t=1$ and $t=3$. The circles in each figure show the Merton policy for the investor with no personal savings. It shows how the stock proportions vary over the different market return states. Figure 2a shows the case where the initial ratio of personal savings to wealth is relatively high, with $E_{0}\left(S_{T}\right) / W_{0}=2$. This could be the position of a young person with modest investible wealth and large uncertain personal-savings prospects. In Figure 2b, we show the case where $E_{0}\left(S_{T}\right) / W_{0}=1$. This illustrates the position of a middle-aged person, saving for retirement, who has substantial investible wealth, but who also has future uncertain personal-savings. In Figure 2c, we show the case of a retired investor who has substantial investible wealth, but no future uncertain personal savings. As would be expected, the young person with considerable future income prospects follows the most aggressive portfolio strategy, whereas the retired investor follows the Merton, constant stock proportion strategy. Note that the full portfolio strategy, across personal savings and market return states, for the middle aged person is shown in Table 2 .

\subsection{Comparative Statics: The Effect of Uncertainty and Correlation}

In Figure 3a-3c, we compare the case of medium size, risky personal savings with two further cases, first, where the personal savings has the same size, but no risk and second, where the personal savings has the same risk, but is positively correlated with the market return. In Figure $3 \mathrm{a}$, we show again the stock proportions in the base case, where $E_{0}\left(S_{T}\right) / W_{0}=1$, the 
volatility of personal savings expectations: $\sigma=0.25$, the coefficient of relative risk aversion: $r=2$, and the correlation of the personal savings information process and the market return: $\rho=0$. We then assume, in Figure 3b, that the personal savings process has no risk, i.e. that $S_{T}=100$ for certain. The stock proportions in this case are shown in Table 3 , and the stock proportions are plotted in Figure 3b. Table 3 illustrates the increasing proportional risk aversion effect of Lemma 1 . In the lowest market state at $t=4$, the investor chooses the highest leverage (the highest stock proportion). Comparing Figures $3 \mathrm{a}$ and $3 \mathrm{~b}$, the investor chooses a wider range (across market states) of stock proportions when personal savings is certain. Also, the uncertainty of the personal savings has a much greater effect in the low market return states (those with the highest stock proportions in the Figures) than in the high market return states. Note that the risk of the personal savings has very little effect in the top market return states, but has a radical effect in the low return states.

Figure 3c illustrates the effect of correlation on the portfolio strategy. If the personal savings of the investor is correlated with the market return, the investor can hedge a proportion of the risk by adjusting the investment in stocks and bonds. Even a modest level of correlation ( $\rho=0.2$ in this example) has a significant impact on the optimal allocation. Comparing Figures 3a and 3c, the investor chooses stock proportions with far less variance over the market states. The optimal strategy, shown by the bond proportions in Table 4, is more conservative in general (higher bond proportions) as well as having less variance across both market return and across personal savings states.

\subsection{Comparative Statics: The Effect of Risk Aversion}

Now we examine the effect of risk aversion on the investor's response to uncertain personal savings. Throughout, we assume that $E_{0}\left(S_{T}\right) / W_{0}=2, \sigma=0.25$, the coefficient of relative risk aversion: $r=2$, and $\rho=0$. We then show, in Figures $4 \mathrm{a}-4 \mathrm{c}$, the results for an investor with risk aversion $r=2, r=3$ and $r=5$. With $r=2$, the optimal strategy for an investor without personal savings, is to invest $64 \%$ of investible wealth in stocks. When $r=3$, this proportion drops to $25 \%$ and when $r=5$ to $10 \%$. Correspondingly, the stock proportions in the case of uncertain personal savings are also lower in the cases of higher risk aversion. Also, Figures $4 \mathrm{~b}$ and $4 \mathrm{c}$ show that the optimal strategies are less variable across market return states, than in Figure 4a. Fundamentally, the optimal policy of the highly risk averse investor varies little due to the overwelming dominance of bonds in the strategy. 


\subsection{The Effect of the Rate of Uncertainty Resolution}

The impact of the resolution of the uncertainty of personal-savings on portfolio choice is analysed in the two-period examples, summarised in Table 7. $\alpha$, the proportion of uncertainty resolved over the first period, ranges from $25 \%$ to $75 \%$. We show results for four levels of risk aversion, with $r=20$ included to show effects in the limiting case. In the second period, the results are unambiguous. The more uncertainty has been resolved in the first period, the more tradable risk the investor takes in the second period. In the first period, the results are not so clearcut. For $r=20$ early resolution of uncertainty $(\alpha=0.75)$ leads to a higher stock proportion in the first period. This is also the case when risk aversion is at levels $r=5$ and $r=10$. However, at risk aversion level $r=2$, the results are somewhat ambiguous. In the first period, the investor faced with later resolution of uncertainty does not always take less risk. The conditions for earlier resolution of uncertainty to increase or decrease risk taking in the first period are complex. It appears that in general it could go either way.

\subsection{State-Contingent Claims Demand.}

We now assume a binomial density, $n>1$, and investigate the effect of personal-savings uncertainty. In Table 8, wealth allocations are reported for $t=1$ and $t=2$, for a binomial density: $n=4$. There are five states of the market portfolio at time $t=1$, indexed by the number of down moves of the binomial process, $i=0,1,2,3$, 4 . Similarly, there are five states of the market portfolio at $t=2$ conditional on each state at $t=1$. Similarly, there are five personal-savings states in period 1 and, for each of these, there are five personalsaving states at time $t=2$. In the table, the $t=2$ allocations are those given the central date 1-node for the personal savings, i.e. the state where expectations of personal savings are unchanged. Since there are five possible states after one period, we report the wealth allocation on a state-by-state basis. This cannot be summarised by a stock proportion at time $t=0$, since the payoff function is no longer linear in this case. The top half of the table shows the optimal wealth allocations, in the absence of personal-savings uncertainty, at time $t=1$ and then at time $t=2$, conditional on states $0,1,2,3,4$ at $t=1$. The bottom half of the table shows the allocations when personal-saving uncertainty is introduced. Comparing the allocations for $t=1$, the investor reacts to personal-savings uncertainty by purchasing more claims on the lowest market state $(71 v 62)$, financing the purchase by reducing claims on the highest market state $(158 v 170)$. A similar effect is shown by the allocations in period 2 . 


\subsection{Long-Term Portfolio Strategy When Borrowing is Constrained}

The simulations described so far assume that borrowing is unconstrained. The solution method guarantees that the first order conditions for a maximum hold at each time and in each state. It could be argued that some of the results are unrealistic, requiring high personal leverage in those states where the tradable wealth is low and where expected personal savings are high. Naturally, in these states, the investor has an incentive to borrow heavily against his future savings. The results indicate an optimal strategy that might be difficult or impossible to put into effect. In this section we investigate the effect of a simple borrowing constraint. We assume simply that the investor can only borrow up to a given proportion of his tradable wealth. The effects of the constraint are illustrated in Table 9 . Table 1 shows the unconstrained borrowing solution for the base case. Tables 9a, 9b, and 9c then show the effects of borrowing constraints of $100 \%, 50 \%$, and $0 \%$ of investible wealth, respectively.

Since the personal leverage in the unconstrained optimisation exceeds $50 \%$ in a number of states, the constrained optimisation in Table $9 \mathrm{~b}$ shows that the constraint is effective in many states, with the investor using leverage of up to $50 \%$. Note also, that in the optimal constrained solution, the investor uses less leverage in the earlier periods when the constraint is not binding. This is to be expected given Lemma 2 and $r>1$. The investor knows that he will be following a more conservative strategy in the later periods and will require less claims in the extreme states. In Table 9d these results are confirmed for the case of a $100 \%$ borrowing constraint. Finally, in Table 9c, where the investor cannot borrow at all, the optimal strategy is a $100 \%$ investment in stocks in most states.

\subsection{Long-Term Portfolio Strategy: Horizon Effects}

Table 10 shows the results of a simulation over ten periods. This shows a little more clearly how portfolio strategy changes over the lifetime of an investor as the horizon date, on which he can access his personal savings, approaches. In this example, where the risk of

personal savings is moderately risky, the investor with ten years to retirement invests $11 \%$ of tradable wealth in bonds and $89 \%$ in stocks. As time goes by, the strategy changes both with realisations of the market return and with changes in the expectations of personal savings. However in the middle states (where $i=j=t / 2$ ), where personal-savings expectations are unchanged and the market return is neither extremely large or small, the stock proportion remains at approximately $89 \%$. This illustrates the Proposition 3 conclusion that there are offsetting effects of the positive drift of the market portfolio and the reduction in the risk of personal savings. 


\section{Conclusions}

We have studied a model, where an investor has utility for terminal wealth, and where the extent of personal savings out of bequests, sales of properties, labor income, and other sources, is revealed over time up to the terminal date. The model is relevant for the common case where an investor invests in a pension plan.

We have shown that the optimal portfolio strategies of investors can be affected quite substantially by personal-savings risk. The key variable affecting strategy is the ratio of investible wealth to expected personal savings. This ratio changes over time as the market return unfolds and as information is revealed about the size of personal savings.

The existence of non-stochastic personal savings causes the indirect utility function to be less risk averse and to exhibit increasing relative risk aversion. The investor buys more stock, substituting personal savings for bond income. However, when personal savings are risky and (perhaps) correlated with the market portfolio, the effect on portfolio policy is to make investors more risk averse and to demand portfolio insurance. The conclusions, known from a single period analysis of the response to non-unhedgeable background risk, apply in a multiperiod world where portfolios are adjusted dynamically over time.

An older investor tends to choose a lower stock proportion than a younger one if his ratio of tradable wealth over expected personal savings is higher. But his residual uncertainty about savings also tends to be lower which induces him to raise his stock proportion. The more is uncertainty resolved early, the more agressive is the portfolio policy, especially in later periods. However, this is not necessarily the case in the earlier periods. Borrowing constraints tend to reduce the risk taking of an investor with constant relative risk aversion above unity.

The analysis illustrates the importance of non-hedgeable background risk on financial decision making. Conclusions based on models with risk-free personal savings need to be modified and may be reversed, when savings are risky. Also, optimal strategies are complex and state dependent.

We have assumed that future risk-free rates of interest are non-stochastic. Also, we have not analysed models where the stock market return is mean reverting. Also, the assumption

of constant relative risk aversion could be relaxed. These and other extensions must await further research. 


\section{Appendix}

\subsection{Proof of Proposition 2}

We have to prove that, given a state at date $t$, the optimal portfolio return $R_{t, t+1}\left(\phi_{t, t+1}\right)$ is less sloped everywhere if $S_{t}=E_{t}\left(S_{T}\right)$ is given and is random instead of non-random. In a state contingent claims model, this is what is meant by a more conservative strategy.

The investor maximizes $E_{t}\left[u\left(W_{T}+S_{T}\right)\right]$ subject to a budget constraint. At date $t$ his tradable wealth is $W_{t}$. This amount is spent on the purchase of date- $(t+1)$-claims. At date $(t+1)$ the investor invests the amount $W_{t+1}$ in date- $(t+2)$ claims and so on. The same optimal allocation $\left\{W_{T}+S_{T}\right\}$ is obtained if the investor maximizes $E_{t}\left[u\left(w_{T}\right)\right]$ subject to the wealth dynamics:

$$
\begin{aligned}
w_{0} & =W_{0}+S_{0} R_{f}^{-T} \\
w_{t+1} & =w_{t} R_{t, t+1}+\left(S_{t+1}-S_{t}\right) R_{f}^{t+1-T} ; t=0, \ldots, T-1
\end{aligned}
$$

and hence,

$$
w_{T}=W_{T}+S_{T} .
$$

The only difference between this and the original model is that at date 0 the investor borrows $S_{0}$ discounted at the risk-free rate against his personal savings and subsequently at every date up to $(T-1)$ adjusts his borrowing to the updated savings expectation by borrowing, in addition, $\left(S_{t+1}-S_{t}\right) R_{f}^{t+1-T}$. In a perfect market this is always possible and has no effects on the optimal allocation. Note that, given the difference between $w_{t}$ and $W_{t}$, the return $R_{t, t+1}$ on $w_{t}$ differs from that on $W_{t}$. We use this model to prove Proposition 2.

The proof proceeds as follows: We first show that the optimal portfolio return $R_{t, t+1}$ is linearly increasing in $\phi_{t, t+1}^{-1 / r}$, if no savings risk exists. We then show that with savings risk the return $R_{t, t+1}$ is convex in $\phi_{t, t+1}^{-1 / r}$. Finally, we show that for $\phi_{t, t+1}^{-1 / r} \rightarrow \infty$ the slope $\partial R_{t, t+1} / \partial \phi_{t, t+1}^{-1 / r} \rightarrow c$ and $\mathrm{c}$ is smaller than the slope without savings risk. This and convexity imply that the slope with savings risk is smaller everywhere than the slope without savings risk and the return curves with and without savings risk intersect once.

\section{$\underline{\text { Step } 1}$}

First, we show that the return function $R_{t, t+1}\left(\phi_{t, t+1}^{-1 / r}\right)$ is linear without savings risk. In this case $S_{t}=S_{T} \cdot \phi_{t, t+1}$ is assumed to be i.i.d. and the investor maximizes $(1-r)^{-1} E_{t}\left(w_{T}^{1-r}\right)=$ 
$(1-r)^{-1} E_{t}\left[\left(w_{t} R_{t, t+1} \cdots R_{T-1, T}\right)^{1-r}\right]$. Since the future investment opportunity set is nonrandom, the optimal policy is myopic. Hence, at date $t$, the investor maximizes $(1-$ $r)^{-1} E_{t}\left[\left(w_{t} R_{t, t+1}\right)^{1-r}\right]$ s.t. $E_{t}\left(R_{t, t+1} \phi_{t, t+1}\right)=1$. The first order condition is ${ }^{9}$

$$
w_{t}^{1-r} R_{t, t+1}^{-r}=\lambda_{t} \phi_{t, t+1}
$$

where $\lambda_{t}$ is the Lagrange-multiplier. Hence $R_{t, t+1}$ is linearly increasing in $\phi_{t, t+1}^{-1 / r}$.

Step 2 We now analyse the optimal portfolio policy in the last period. Let $s_{T} \equiv S_{T}-S_{T-1}$ so that $E_{T-1}\left(s_{T}\right)=0$. The investor maximizes

$$
\begin{aligned}
E_{T-1}\left[u\left(w_{T}\right)\right] & =(1-r)^{-1} E_{T-1}\left[\left(w_{T-1} R_{T-1, T}+s_{T}\right)^{1-r}\right] \\
\text { s.t. } 1 & =E_{T-1}\left(R_{T-1, T} \phi_{T-1, T}\right) .
\end{aligned}
$$

Using Kimball's concept of the precautionary premium, the first order condition can be written as

$$
\begin{aligned}
E_{T-1}\left[\left(w_{T-1} R_{T-1, T}+s_{T}\right)^{-r} \mid \phi_{T-1, T}\right] & =\left[w_{T-1} R_{T-1, T}-\varphi_{T}\right]^{-r} \\
& =\frac{\lambda_{T-1}}{w_{T-1}} \phi_{T-1, T}
\end{aligned}
$$

The precautionary premium $\varphi_{T}$ is the subtracted non-random amount which yields the same expected marginal utility as the random amount $s_{T} \cdot \varphi_{T}$ is a function of $w_{T-1} R_{T-1, T}$. In FSS (1998) it is shown, given $w_{T-1}$, that $\varphi_{T}>0, \partial \varphi_{T} / \partial R_{T-1, T}<0$ and $\partial^{2} \varphi_{T} / \partial R_{T-1, T}^{2}>0$. For $R_{T-1, T} \rightarrow \infty, \varphi_{T} \rightarrow 0$.

Rewrite equation (13) as

$$
w_{T-1} R_{T-1, T}-\varphi_{T}=\left(\frac{\lambda_{T-1}}{w_{T-1}}\right)^{-1 / r} \phi_{T-1, T}^{-1 / r}
$$

Given $w_{T-1}$, from the convexity of $\varphi_{T}$ in $R_{T-1, T}$ and (14) it follows immediately that $R_{T-1, T}\left(\phi_{T-1, T}^{-1 / r}\right)$ is convex.

\footnotetext{
${ }^{9}$ To simplify notation, we drop "*" in $R_{T-1, T}^{*}$.
} 
For $\phi_{T-1, T}^{-1 / r} \rightarrow \infty, R_{T-1, T} \rightarrow \infty$, and $\varphi_{T} \rightarrow 0$. Hence $\partial R_{T-1, T} / \partial \phi_{T-1, T}^{-1 / r} \rightarrow\left(\lambda_{T-1} / w_{T-1}^{1-r}\right)^{-1 / r}$. This slope is smaller with than without savings risk if $\lambda_{T-1}^{-1 / r}$ is smaller since $w_{T-1}^{1-r}$ is the same. Multiply equation (14) by $\phi_{T-1, T}$ and take expectations. This yields

$$
w_{T-1}-E_{T-1}\left[\phi_{T-1, T} \varphi_{T}\right]=\left(\frac{\lambda_{T-1}}{w_{T-1}}\right)^{-1 / r} E_{T-1}\left(\phi_{T-1, T}^{1-1 / r}\right)
$$

Hence, given $w_{T-1}$, the precautionary premium lowers $\lambda_{T-1}^{-1 / r}$. Therefore, for $\phi_{T-1, T}^{-1 / r} \rightarrow \infty$ the slope is smaller with than without savings risk. This and convexity imply that the slope is smaller everywhere. This proves Proposition 2 for the final period.

Step 3

Third, we now prove Proposition 2 for period $(T-2, T-1)$.

$$
\begin{aligned}
W_{T}+S_{T} & =w_{T-1} R_{T-1, T}+s_{T} \\
& =\left(w_{T-2} R_{T-2, T-1}+s_{T-1} / R_{f}\right) R_{T-1, T}+s_{T} \text { with } s_{T-1} \equiv S_{T-1}-S_{T-2} .
\end{aligned}
$$

The investor maximizes

$$
\begin{aligned}
E_{T-2}\left[u\left(W_{T}+S_{T}\right)\right] & =(1-r)^{-1} E_{T-2}\left[\left(W_{T}+S_{T}\right)^{1-r}\right] \\
\text { s.t. } 1 & =E_{T-2}\left(R_{T-2, T} \phi_{T-2, T-1}\right)
\end{aligned}
$$

given $w_{T-2}$ and given an optimal policy $R_{T-1, T}$. The first order condition is

$$
\begin{aligned}
& E_{T-2} E_{T-1}\left[\left(\left(w_{T-2} R_{T-2, T-1}+s_{T-1} / R_{f}\right) R_{T-1, T}+s_{T}\right)^{-r} R_{T-1, T} \mid \phi_{T-2, T-1}\right] \\
= & \frac{\lambda_{T-2}}{w_{T-2}} \phi_{T-2, T-1}
\end{aligned}
$$

since $\partial R_{T-1, T} / \partial\left(w_{T-1} R_{T-2, T-1}\right)$ drops out as explained before equation (10). 
Consider some state at date $(T-1)$ with superscript $o$. Then it follows from equation (13) that the marginal rate of substitution for this and some other state equals the ratio of prices for state-contingent claims, i.e.

$$
\begin{aligned}
& E_{T-1}\left[\left(w_{T-1} R_{T-1, T}+s_{T}\right)^{-r} \mid \phi_{T-1, T}\right] \\
= & E_{T-1}\left[\left(w_{T-1} R_{T-1, T}^{o}+s_{T}\right)^{-r} \mid \phi_{T-1, T}^{o}\right] \frac{\phi_{T-1, T}}{\phi_{T-1, T}^{o}}
\end{aligned}
$$

Now we specify state $o$ as the crossover-state at date $(T-1)$. Given a state at date $(T-1)$, the investor optimizes his return function $R_{T-1, T}\left(\phi_{T-1, T}\right)$. In the presence of savings risk, the optimal return function depends on the endowment $w_{T-1}$. If $w_{T-1}$ increases marginally by $d w_{T-1}$, the return function changes. Due to the budget constraint there must exist some $\phi_{T-1, T}^{o}\left(w_{T-1}\right)$ at which the return functions for $w_{T-1}$ and for $\left(w_{T-1}+d w_{T-1}\right)$ intersect. We call this the crossover price and $R_{T-1, T}^{o}$ the crossover return. In the following we use this crossover-state since, by definition, $R_{T-1, T}^{o}$ and $\phi_{T-1, T}^{o}$ are not affected by marginal changes in $w_{T-1}$.

Since $w_{T-1}=w_{T-2} R_{T-2, T-1}+s_{T-1} / R_{f}$, equation (16) yields

$$
\begin{aligned}
& E_{T-1}\left[\left(\left(w_{T-2} R_{T-2, T-1}+s_{T-1} / R_{f}\right) R_{T-1, T}+s_{T}\right)^{-r} \mid \phi_{T-1, T}\right] \\
= & E_{T-1}\left[\left(\left(w_{T-2} R_{T-2, T-1}+s_{T-1} / R_{f}\right) R_{T-1, T}^{o}+s_{T}\right)^{-r} \mid \phi_{T-1, T}^{o}\right] \frac{\phi_{T-1, T}}{\phi_{T-1, T}^{o}} .
\end{aligned}
$$

Insert this in equation (15) and obtain

$$
\begin{aligned}
& E_{T-2} E_{T-1}\left[\left(\left(w_{T-2} R_{T-2, T-1}+s_{T-1} / R_{f}\right) R_{T-1, T}^{o}+s_{T}\right)^{-r} \frac{R_{T-1, T} \phi_{T-1, T}}{\phi_{T-1, T}^{o}} \mid \phi_{T-2, T-1}\right] \\
= & \frac{\lambda_{T-2}}{w_{T-2}} \phi_{T-2, T-1} .
\end{aligned}
$$

From the budget constraint $E_{T-1}\left(R_{T-1, T} \phi_{T-1, T}\right)=1$. Hence we can rewrite (18) after factoring out $w_{T-2}$ and $R_{T-1, T}^{o}$

$$
E_{T-2}\left[\frac{\left(R_{T-1, T}^{o}\right)^{-r}}{\phi_{T-1, T}^{o}} E_{T-1}\left[\left(R_{T-2, T-1}+\frac{s_{T-1}}{w_{T-2} R_{f}}+\frac{s_{T}}{w_{T-2} R_{T-1, T}^{o}}\right)^{-r}\right] \mid \phi_{T-2, T-1}\right]
$$




$$
=\frac{\lambda_{T-2}}{w_{T-2}^{1-r}} \phi_{T-2, T-1} .
$$

Now divide equation (19) by $E_{T-2}\left(\left(R_{T-1, T}^{o}\right)^{-r} / \phi_{T-1, T}^{o}\right) \equiv z_{T-2}$. Since $\left(\left(R_{T-1, T}^{o}\right)^{-r} / \phi_{T-1, T}^{o}\right)$ $/ z_{T-2}$ is positive and has expectation $E_{T-2}(\cdot)=1, E_{T-2}\left(\left(R_{T-1, T}^{o}\right)^{-r} /\left(\phi_{T-1, T}^{o} z_{T-2}\right)(\cdot)\right)$ is the expectation under an equivalent probability measure $\mathrm{Q}$, for period $t-2$ to $t-1$. Therefore we can restate (19) as

$$
\begin{aligned}
& E_{T-2}^{Q}\left[E_{T-1}\left[\left(R_{T-2, T-1}+\frac{s_{T-1}}{w_{T-2} R_{f}}+\frac{s_{T}}{w_{T-2} R_{T-1, T}^{o}}\right)^{-r}\right] \mid \phi_{T-2, T-1}\right] \\
= & \frac{\lambda_{T-2}}{w_{T-2}^{1-r} z_{T-2}} \phi_{T-2, T-1} .
\end{aligned}
$$

Under this equivalent probability measure $E_{T-2}^{Q}\left(s_{T-1}\right) \neq 0$. Therefore in order to use the precautionary premium, define $s_{T-1}^{Q} \equiv s_{T-1}-E_{T-2}^{Q}\left(s_{T-1}\right)$. Then (20) yields

$$
\begin{aligned}
& E_{T-2}^{Q}\left[E_{T-1}\left[\left(R_{T-2, T-1}+\frac{E_{T-2}^{Q}\left(s_{T-1}\right)+s_{T-1}^{Q}}{w_{T-2} R_{f}}+\frac{s_{T}}{w_{T-2} R_{T-1, T}^{o}}\right)^{-r}\right] \mid \phi_{T-2, T-1}\right] \\
= & \frac{\lambda_{T-2}}{w_{T-2}^{1-r} z_{T-2}} \phi_{T-2, T-1} .
\end{aligned}
$$

Define $y_{T-1} \equiv \frac{s_{T-1}^{Q}}{w_{T-2} R_{f}}+\frac{s_{T}}{w_{T-2} R_{T-1, T}^{o}}$. Then rewrite $(21)$ as

$$
\begin{aligned}
& E_{T-2}^{Q}\left[\left(R_{T-2, T-1}+\frac{E_{T-2}^{Q}\left(s_{T-1}\right)}{w_{T-2} R_{f}}+y_{T-1}\right)^{-r} \mid \phi_{T-2, T-1}\right] \\
= & \frac{\lambda_{T-2}}{w_{T-2}^{1-r} z_{T-2}} \phi_{T-2, T-1}
\end{aligned}
$$

As $E_{T-1}\left(s_{T}\right)=0$ and $E_{T-2}^{Q}\left(s_{T-1}^{Q}\right)=0, E_{T-2}^{Q}\left[y_{T-1}\right]=0$. This enables us to use the precautionary premium $\varphi^{Q}=\varphi^{Q}\left(y_{T-1}\right)$ based on the equivalent probability measure so that (22) yields 


$$
\left[R_{T-2, T-1}+\frac{E_{T-2}^{Q}\left(s_{T-1}\right)}{w_{T-2} R_{f}}-\varphi^{Q}\right]^{-r}=\frac{\lambda_{T-2}}{w_{T-2}^{1-r} z_{T-2}} \phi_{T-2, T-1}
$$

or

$$
R_{T-2, T-1}+\frac{E_{T-2}^{Q}\left(s_{T-1}\right)}{w_{T-2} R_{f}}-\varphi^{Q}=\left(\frac{\lambda_{T-2}}{w_{T-2}^{1-r} z_{T-2}}\right)^{-1 / r} \phi_{T-2, T-1}^{-1 / r} .
$$

Note that a marginal change in $R_{T-2, T-1}$ does not change the probability measure $\mathrm{Q}$, since it does not change the crossover-state at date $(T-1)$.

Since $\partial E_{T-2}^{Q}\left(s_{T-1}\right) / \partial R_{T-2, T-1}=0$ and $\partial \varphi^{Q} / \partial R_{T-2, T-1}<0$ and $\partial^{2} \varphi^{Q} / \partial R_{T-2, T-1}^{2}>0$, differentiating (23) twice with respect to $\phi_{T-2, T-1}^{-1 / r}$ immediately shows that $R_{T-2, T-1}$ is convex in $\phi_{T-2, T-1}^{-1 / r}$.

It remains to be shown that $\partial R_{T-2, T-1} / \partial \phi_{T-2, T-1}^{-1 / r}$ for $\phi_{T-2, T-1}^{-1 / r} \rightarrow \infty$ is smaller in the case of savings risk. For $\phi_{T-2, T-1}^{-1 / r} \rightarrow \infty, R_{T-2, T-1} \rightarrow \infty$ so that the impact of savings risk $s_{T-1}$ and $s_{T}$ in the first order condition (15) becomes negligible. Hence for $\phi_{T-2, T-1}^{-1 / r} \rightarrow \infty$, equation (15) simplifies to

$$
E_{T-1}\left[\left(w_{T-2} R_{T-2, T-1} R_{T-1, T}\right)^{-r} R_{T-1, T} \mid \phi_{T-2, T-1}\right]=\frac{\lambda_{T-2}}{w_{T-2}} \phi_{T-2, T-1} .
$$

This is the same as the first order condition without savings risk. $R_{T-1, T}\left(\phi_{T-1, T}\right)$ is also independent of $R_{T-2, T-1}$. Hence the slope $\partial R_{T-2, T-1} / \partial \phi_{T-2, T-1}^{-1 / r}$ is smaller in the case of savings risk if $\lambda_{T-2}^{-1 / r}$ is smaller. We prove this by using

$$
E_{T-2}\left(\frac{\lambda_{T-1}}{w_{T-1}}\right)=\frac{\lambda_{T-2}}{R_{f} w_{T-2}}
$$

Since savings risk raises $\lambda_{T-1} / w_{T-1} \forall w_{T-1}$, by (24) $\lambda_{T-2}$ increases, too, given $w_{T-2}$. This holds even though savings risk changes $R_{T-2, T-1}\left(\phi_{T-2, T-1}\right)$.

In order to derive (24), consider equation (15) and note that $w_{T-2} R_{T-2, T-1}+s_{T-1} / R_{f}=$ $w_{T-1}$. Hence we can insert equation (13) in equation (15) and obtain

$$
E_{T-2}\left[\frac{\lambda_{T-1}}{w_{T-1}} R_{T-1, T} \phi_{T-1, T} \mid \phi_{T-2, T-1}\right]=\frac{\lambda_{T-2}}{w_{T-2}} \phi_{T-2, T-1} .
$$


Due to the budget constraint $E_{T-1}\left[R_{T-1, T} \phi_{T-1, T}\right]=1$. Taking expectations across $\phi_{T-2, T-1}$ yields (24). This proves Proposition 2 for the period $(T-2, T-1)$. The proof is the same for the earlier periods. 


\subsection{Proof of Lemma 2}

The investor maximises $E_{t}\left[u\left(w_{t}\right)\right]$ subject to the wealth dynamics $w_{0}=W_{0}+S_{0} R_{f}^{-T}$ and $w_{t+1}=w_{t} R_{t, t+1}$, given non-stochastic personal savings. Let $R_{t, t+1}$ denote the optimal return in period $t+1$ and let

$$
R_{t, T}=R_{t, t+1} \ldots R_{T-1, T}
$$

The indirect utility of wealth is

$$
J\left(w_{t}\right)=E_{t}\left[u\left(w_{t} R_{t, T}\right)\right]=\frac{1}{1-r} E_{t}\left[\left(w_{t} R_{t, T}\right)^{1-r}\right] .
$$

Differentiate with respect to $w_{t}$ to obtain

$$
J^{\prime}\left(w_{t}\right)=E_{t}\left[w_{t}^{-r} R_{t, T}^{1-r}\right]=\frac{1-r}{w_{t}} J\left(w_{t}\right) .
$$

Note that the term $\partial R_{t, T} / \partial w_{t}$ drops out as explained before equation (10).

If a borrowing constraint is imposed and becomes binding at date $t$ and or subsequently, then $J\left(w_{t}\right)$ is reduced. Hence $J^{\prime}\left(w_{t}\right)$ increases [declines] if $r>[<] 1$ and stays constant for $r=1$.

Consider two states $u$ and $d$ at date $t+1$. Then the IOC yields

$$
\frac{J^{\prime}\left(w_{t+1}^{u}\right)}{J^{\prime}\left(w_{t+1}^{d}\right)}=\frac{\phi_{t, t+1}^{u}}{\phi_{t, t+1}^{d}},
$$

where the superscripts denote the variables for the respective states. From (25)

$$
\begin{aligned}
\frac{J^{\prime}\left(w_{t+1}^{u}\right)}{J^{\prime}\left(w_{t+1}^{d}\right)} & =\left(\frac{w_{t+1}^{u}}{w_{t+1}^{d}}\right)^{-r} \frac{E_{t+1}^{u}\left(R_{t+1, T}^{1-r}\right)}{E_{t+1}^{d}\left(R_{t+1, T}^{1-r}\right)} \\
& =\left(\frac{R_{t, t+1}^{u}}{R_{t, t+1}^{d}}\right)^{-r} \frac{E_{t+1}^{u}\left(R_{t+1, T}^{1-r}\right)}{E_{t+1}^{d}\left(R_{t+1, T}^{1-r}\right)} .
\end{aligned}
$$

Let $r>1$. Then the effect of the borrowing constraint is to raise $J^{\prime}\left(w_{t+1}^{d}\right)$ and hence $E_{t+1}^{d}\left(R_{t+1, T}^{1-r}\right)$, while $J^{\prime}\left(w_{t+1}^{u}\right)$ and hence $E_{t+1}^{u}\left(R_{t+1, T}^{1-r}\right)$ stays constant. Hence, in order to satisfy the IOC (26), $R_{t, t+1}^{d} / R_{t, t+1}^{u}$ has to increase. The proof is the same for $r<1$. For $r=0$, there is no effect since $J^{\prime}\left(w_{t}\right)$ is not affected by the borrowing constraint. 


\subsection{HSS Approximation of a Two-factor Diffusion Process}

Lemma 3 (Approximation of a Two-factor Diffusion Process) Suppose that $X_{t}, Y_{t}$ follows a joint lognormal process, where $E_{0}\left(X_{t}\right)=1, E_{0}\left(Y_{t}\right)=1 \forall t$, and where in discretised form

$$
\begin{aligned}
x_{t} & =\alpha_{x, t}+\beta_{x, t} x_{t-1}+\varepsilon_{x, t} \\
y_{t} & =\alpha_{y, t}+\beta_{y, t} y_{t-1}+\gamma_{y, t} x_{t-1}+\delta_{y, t} x_{t}+\varepsilon_{y, t}
\end{aligned}
$$

Let the conditional logarithmic standard deviation of $j_{t}$ be denoted as $\sigma_{j}(t)$ for $j=(X, Y)$, where

$$
\sigma_{j}^{2}(t)=\operatorname{var}\left(\varepsilon_{\mathrm{j}, \mathrm{t}}\right)
$$

If $j_{t}$ is approximated by a log-binomial distribution with binomial density $N_{t}=N_{t-1}+n_{t}$ and if the proportionate up and down movements, $u_{j_{t}}$ and $d_{j_{t}}$ are given by

$$
\begin{aligned}
& d_{j_{t}}=\frac{2}{1+\exp \left(2 \sigma_{j}(t) \sqrt{\tau_{t} / n_{t}}\right)} \\
& u_{j_{t}}=2-d_{j_{t}}
\end{aligned}
$$

and the conditional probability of an up-move at node $r$ of the lattice is given by

$$
q_{j_{t}}=\frac{E_{t-1}\left(j_{t}\right)-\left(N_{t-1}-r\right) \ln \left(u_{j_{t}}\right)-\left(n_{t}+r\right) \ln \left(d_{j_{t}}\right)}{n_{t}\left[\ln \left(u_{j_{t}}\right)-\ln \left(d_{j_{t}}\right)\right]}
$$

then the unconditional mean and volatility of the approximated process approach their true values, i.e., $\hat{E}_{0}\left(j_{t}\right) \rightarrow 1$ and $\hat{\sigma}_{j_{t}} \rightarrow \sigma_{j_{t}}$ as $n \rightarrow \infty$.

Lemma 3 is proved in Peterson and Stapleton (2002). It allows us to build a two-dimensional, recombining tree of two joint-lognormal variables, each with a unit expectation. The variables can then be scaled, in the present case, to reflect the expected market portfolio return and the expected personal savings. The essential property of the recombining tree methodology is that the number of states does not increase exponentially. This makes computation of optimal policies feasible for problems with a realistic number of time periods. 


\subsection{The Log-linear Approximation in Viceira (2001)}

Following Viceira (2001), upper case variables refer to the natural numbers and lower case variables to logarithms. Equation numbers in Viceira are referred to as $\mathrm{V}($ ). In Viceira (2001) the intertemporal budget constraint, $\mathrm{V}(5)$ is:

$$
W_{t+1}=\left(W_{t}+Y_{t}-C_{t}\right) R_{p, t+1}
$$

Wealth at time $t+1$ equals wealth at time $t+$ labor income at time $t$ - consumption at time $t$ compounded at the portfolio return. The log-linear approximation in $\mathrm{V}(9)$ is:

$$
w_{t+1} \approx k+\rho_{w}\left(w_{t}-y_{t}\right)-\rho_{c}\left(c_{t}-y_{t}\right)+y_{t}+r_{p, t+1}
$$

Substituting for $c_{t}-y_{t}$, using $\mathrm{V}(18)$ :

$$
\begin{gathered}
c_{t}-y_{t}=b_{0}+b_{1}\left(w_{t}-y_{t}\right), \\
w_{t+1} \approx k+\rho_{w}\left(w_{t}-y_{t}\right)-\rho_{c}\left(b_{0}+b_{1}\left(w_{t}-y_{t}\right)\right)+y_{t}+r_{p, t+1} .
\end{gathered}
$$

Taking anti-logs

$$
W_{t+1}=a W_{t}^{\left[\rho_{w}-\rho_{c} b_{1}\right]} Y_{t}^{\left[1-\rho_{w}+\rho_{c} b_{1}\right]} R_{p, t+1},
$$

where $a$ is a constant. In this approximation, wealth at time $t+1$ is the product of the wealth term $W_{t}^{\left[\rho_{w}-\rho_{c} b_{1}\right]}$, which depends on market risk, and a labor income term $Y_{t}^{\left[1-\rho_{w}+\rho_{c} b_{1}\right]}$ which depends on a background risk. Hence, in this model labor income risk is a multiplicative risk. If it is independent of the market risk, it has no effect on the portfolio decisions of a constant relative risk averse investor. 


\section{References}

Balduzzi, P. and Lynch, A.W. (1999), "Transaction Costs and Predictability: Some Utility Cost Calculations", Journal of Financial Economics, 52, 47-78.

Barberis, N. (2000), "Investing for the Long Run when Returns are Predictable", Journal of Finance, 55, 1, 225-264.

Brandt, M.W. (1999), "Estimating Portfolio and Consumption Choice: A Conditional Euler Equations Approach", Journal of Finance, 54, 5, 1609-1645.

Brennan, M.J., Schwartz, E. and Lagnado, R. (1997), "Strategic Asset Allocation", Journal of Economic Dynamics and Control, 21, 1377-1403.

Brennan, M.J. and Xia, Y. (2002), "Dynamic Asset Allocation under Inflation", Journal of Finance, 57, 1201-1238.

Campbell, J. and Viceira, L.M. (2002), Strategic Asset Allocation - Portfolio Choice for Long-Term Investors, Oxford Univ. Press.

Cox, J.C. and Huang, C. (1989), "Optimal Consumption and Portfolio Policies when Asset Prices Follow a Diffusion Process", Journal of Economic Theory, 49, 33-83.

Detemple, J., Garcia, R. and Rindisbacher, M. (2000), "A Monte-Carlo Method for Optimal Portfolios", Cirano Working paper, 1.1.2000.

Elmendorf, D.W. and Kimball, M.S. (2000), "Taxation of Labor Income and the Demand for Risky Assets", International Economic Review, 41, 801-833.

Franke, G., Stapleton, R.C. and Subrahmanyam, M.G. (1998), "Who Buys and Who Sells Options: The Role of Options in an Economy with Background Risk", Journal of Economic Theory, 82, 89-109.

Gollier, C. (2001) The Economics of Risk and Time, MIT press, Cambridge (Mass).

Gollier, C. and Pratt, J. W. (1996), "Weak Proper Risk Aversion and the Tempering Effect of Background Risk", Econometrica, 64, 1109-1123.

Heaton, J. and Lucas, D. (1997), "Market Frictions, Savings Behaviour and Portfolio Choice", Macroeconomic Dynamics, 1, 76-101.

Ho, T.S., Stapleton, R.C. and Subrahmanyam, M.G. (1995), "Multivariate Binomial Approximations for Asset Prices with Non-Stationary Variance and Covariance Characteristics", Review of Financial Studies, 8, 1125-1152. 
Kandel, S. and Stambaugh, R.F. (1996), "On the predictability of stock returns: An asset-allocation perspective", Journal of Finance, 51, 385-424.

Kim, T.S. and Omberg, E. (1996), "Dynamic Nonmyopic Portfolio Behaviour", Review of Financial Studies, 9, 141-161.

Kihlstrom, R.E., Romer, D. and Williams, S. (1981), "Risk Aversion with Random Initial Wealth", Econometrica, 49, 911-920.

Kimball, M.S. (1990), "Precautionary Saving in the Small and in the Large", Econometrica, 58, 53-73.

Kimball, M.S. (1993), "Standard Risk Aversion", Econometrica, 61, 589-611.

Koo, H.K. (1998), "Consumption and Portfolio Selection with Labor Income: A Continuous Time approach", Mathematical Finance, 8, 49-65.

Lynch, A.W. (2001), "Portfolio choice and equity characteristics: characterizing the hedging demands induced by return predictability", Journal of Financial Economics, $62,67-130$.

Merton, R. (1969), "Lifetime Portfolio selection Under Uncertainty", Review of Economics and Statistics, 51, 247-257.

Nachman, D.C. (1982), "Preservation of 'More Risk Averse' under Expectations", Journal of Economic Theory, 28, 361-368.

Nelson, D.B. and Ramaswamy, K. (1990), "Simple Binomial Processes as Diffusion Approximations in Financial Models", Review of Financial Studies, 3, 393-430.

Peterson, S.J. and Stapleton, R.C. (2002), "The Pricing of Bermudan Style-Options on Correlated Assets", Review of Derivatives Research, 5, 127-151

Pratt, J.W. and Zeckhauser, R.J. (1987), "Proper Risk Aversion", Econometrica, 55, 143-154.

Viceira, L.M. (2001) "Optimal Portfolio Choice for Long-horizon Investors with Nontradable Labor Income", Journal of Finance, 56, 433-470.

Xia, Y. (2001), "Learning about Predictability: The Effects of Parameter Uncertainty on Dynamic Asset Allocation", Journal of Finance, 56, 205-246. 


\section{Table 1: Stock Proportions: Equal Resolution of Uncertainty Large, Positive, Risky Personal Savings}

\begin{tabular}{|c|c|c|c|c|c|}
\hline state & Stocks $t=0$ & Stocks $t=1$ & Stockst $=2$ & Stocks $t=3$ & Stocks $t=4$ \\
\hline 0,0 & 1.30 & 1.31 & 1.32 & 1.34 & 1.34 \\
\hline 0,1 & & 1.09 & 1.10 & 1.12 & 1.14 \\
\hline 0,2 & & & 0.95 & 0.96 & 0.98 \\
\hline 0,3 & & & & 0.85 & 0.86 \\
\hline 0,4 & & & & & 0.79 \\
\hline 1,0 & & 1.67 & 1.63 & 1.63 & 1.61 \\
\hline 1,1 & & 1.32 & 1.33 & 1.34 & 1.35 \\
\hline 1,2 & & & 1.10 & 1.11 & 1.13 \\
\hline 1,3 & & & & 0.94 & 0.96 \\
\hline 1,4 & & & & & 0.85 \\
\hline 2,0 & & & 2.48 & 2.22 & 2.06 \\
\hline 2,1 & & & 1.73 & 1.69 & 1.66 \\
\hline 2,2 & & & 1.35 & 1.36 & 1.36 \\
\hline 2,3 & & & & 1.10 & 1.12 \\
\hline 2,4 & & & & & 1.06 \\
\hline 3,0 & & & & 4.63 & 3.26 \\
\hline 3,1 & & & & 2.62 & 2.32 \\
\hline 3,2 & & & & 1.80 & 1.75 \\
\hline 3,3 & & & & 1.38 & 1.38 \\
\hline 3,4 & & & & & 1.11 \\
\hline 4,0 & & & & & 19.75 \\
\hline 4,1 & & & & & 5.22 \\
\hline 4,2 & & & & & 2.82 \\
\hline 4,3 & & & & & 1.88 \\
\hline 4,4 & & & & & 1.42 \\
\hline
\end{tabular}

1. The market return is i.i.d. with expected value of $11 \%$ and volatility of $20 \%$. The one-period risk-free rate is (1/0.95)-1 and non-stochastic.

2. The date 0 -expected value of the personal savings is $E_{0}\left(S_{T}\right)=200$ with volatility $\sigma_{L}=0.25$. Initial investible wealth is $W_{0}=100$.

3. The coefficient of relative risk aversion is $r=2$.

4. Column 1 shows the state $i, j$, where $i$ is the number of down-moves in the market return process and $j$ is the number of down-moves in the personal savings process.

5. Columns 2-6 show the proportion of wealth invested in stocks in the various states. 


\section{Table 2: Stock Proportions: Equal Resolution of Uncertainty Medium Size, Positive, Risky Personal Savings}

\begin{tabular}{|c|c|c|c|c|c|}
\hline state & Stocks $t=0$ & Stocks $t=1$ & Stocks $t=2$ & Stocks $t=3$ & Stocks $t=4$ \\
\hline 0,0 & 1.03 & 1.04 & 1.06 & 1.08 & 1.09 \\
0,1 & & 0.91 & 0.92 & 0.94 & 0.95 \\
0,2 & & & 0.83 & 0.84 & 0.85 \\
0,3 & & & & 0.77 & 0.78 \\
0,4 & & & & & 0.73 \\
\hline 1,0 & & 1.20 & 1.21 & 1.23 & 1.25 \\
1,1 & & 1.01 & 1.03 & 1.05 & 1.06 \\
1,2 & & & 0.90 & 0.91 & 0.92 \\
1,3 & & & & 0.82 & 0.83 \\
1,4 & & & 1.51 & 1.50 & 0.76 \\
\hline 2,0 & & & 1.19 & 1.20 & 1.49 \\
2,1 & & & & 1.02 & 1.03 \\
2,2 & & & & 0.88 & 0.90 \\
2,3 & & & & 2.12 & 0.81 \\
2,4 & & & & 1.50 & 1.99 \\
\hline 3,0 & & & & 0.98 & 1.49 \\
3,1 & & & & & 1.00 \\
3,2 & & & & & 0.87 \\
3,3 & & & & & 3.65 \\
3,4 & & & & & 1.49 \\
\hline 4,0 & & & & & 0.97 \\
4,1 & & & & & \\
4,2 & & & & & \\
4,3 & & & & & \\
4,4 & & & & & \\
\hline
\end{tabular}

1. The market return is i.i.d. with expected value of $11 \%$ and volatility of $20 \%$. The one-period risk-free rate is (1/0.95)-1 and non-stochastic.

2. The date 0 -expected value of the personal savings is $E_{0}\left(S_{T}\right)=100$ with volatility $\sigma_{L}=0.25$. Initial investible wealth is $W_{0}=100$.

3. The coefficient of relative risk aversion is $r=2$.

4. Column 1 shows the state $i, j$, where $i$ is the number of down-moves in the market return process and $j$ is the number of down-moves in the personal savings process.

5. Columns 2-6 show the proportion of wealth invested in stocks in the various states. 


\section{Table 3: Stock Proportions: Equal Resolution of Uncertainty Medium Size, Risk-Free Personal Savings}

\begin{tabular}{|c|c|c|c|c|c|}
\hline state & Stocks $t=0$ & Stocks $t=1$ & Stocks $t=2$ & Stocks $t=3$ & Stocks $t=4$ \\
\hline 0,0 & 1.18 & 1.05 & 0.97 & 0.91 & 0.86 \\
0,1 & & 1.05 & 0.97 & 0.91 & 0.86 \\
0,2 & & & 0.97 & 0.91 & 0.86 \\
0,3 & & & & 0.91 & 0.86 \\
0,4 & & & & & 0.86 \\
\hline 1,0 & & 1.29 & 1.13 & 1.02 & 0.95 \\
1,1 & & 1.29 & 1.13 & 1.02 & 0.95 \\
1,2 & & & 1.13 & 1.02 & 0.95 \\
1,3 & & & & 1.02 & 0.95 \\
1,4 & & & 1.44 & 1.23 & 0.95 \\
\hline 2,0 & & 1.44 & 1.23 & 1.09 \\
2,1 & & & & 1.23 & 1.09 \\
2,2 & & & & 1.23 & 1.09 \\
2,3 & & & & 1.64 & 1.09 \\
2,4 & & & & 1.66 & 1.36 \\
\hline 3,0 & & & & 1.66 & 1.36 \\
3,1 & & & & & 1.36 \\
3,2 & & & & & 2.36 \\
3,3 & & & & & 2.00 \\
3,4 & & & & & 2.00 \\
\hline 4,0 & & & & & \\
4,1 & & & & & \\
4,2 & & & & & \\
4,3 & & & & & \\
4,4 & & & & & \\
\hline
\end{tabular}

1. The market return is i.i.d. with expected value of $11 \%$ and volatility of $20 \%$. The one-period risk-free rate is (1/0.95)-1 and non-stochastic.

2. The date 0 -expected value of the personal savings is $E_{0}\left(S_{T}\right)=100$ with volatility $\sigma_{L}=0$. Initial investible wealth is $W_{0}=100$.

3. The coefficient of relative risk aversion is $r=2$.

4. Column 1 shows the state $i, j$, where $i$ is the number of down-moves in the market return process and $j$ is the number of down-moves in the personal savings process.

5. Columns 2-6 show the proportion of wealth invested in stocks in the various states. 


\section{Table 4: Stock Proportions: Equal Resolution of Uncertainty Medium Size, Correlated Risky Personal Savings}

\begin{tabular}{|c|c|c|c|c|c|}
\hline state & Stocks $t=0$ & Stockst $=1$ & Stocks $t=2$ & Stocks $t=3$ & Stocks $t=4$ \\
\hline 0,0 & 0.88 & 0.90 & 0.92 & 0.94 & 0.96 \\
0,1 & & 0.82 & 0.83 & 0.84 & 0.86 \\
0,2 & & & 0.77 & 0.78 & 0.79 \\
0,3 & & & & 0.73 & 0.74 \\
0,4 & & & & & 0.71 \\
\hline 1,0 & & 0.98 & 1.00 & 1.03 & 1.06 \\
1,1 & 0.87 & 0.89 & 0.91 & 0.93 \\
1,2 & & & 0.81 & 0.82 & 0.83 \\
1,3 & & & & 0.76 & 0.77 \\
1,4 & & & 1.16 & 1.18 & 0.73 \\
\hline 2,0 & & 0.97 & 1.00 & 1.21 \\
2,1 & & & & 0.88 & 0.90 \\
2,2 & & & & 0.80 & 0.81 \\
2,3 & & & & 1.47 & 0.75 \\
2,4 & & & & 1.14 & 1.47 \\
\hline 3,0 & & & & 0.96 & 0.98 \\
3,1 & & & & & 0.87 \\
3,2 & & & & & 0.79 \\
3,3 & & & & & 2.11 \\
3,4 & & & & & 1.45 \\
\hline 4,0 & & & & & 0.95 \\
4,1 & & & & & \\
4,2 & & & & & \\
4,3 & & & & & \\
4,4 & & & & & \\
\hline
\end{tabular}

1. The market return is i.i.d. with expected value of $11 \%$ and volatility of $20 \%$. The one-period risk-free rate is (1/0.95)-1 and non-stochastic.

2. The date 0 -expected value of the personal savings is $E_{0}\left(S_{T}\right)=100$ with volatility $\sigma_{L}=0.25$. Initial investible wealth is $W_{0}=100$. The correlation between the market return and personal savings processes is $\rho=0.2$.

3. The coefficient of relative risk aversion is $r=2$.

4. Column 1 shows the state $i, j$, where $i$ is the number of down-moves in the market return process and $j$ is the number of down-moves in the personal savings process.

5. Columns 2-6 show the proportion of wealth invested in stocks in the various states. 
Table 5: Stock Proportions: Equal Resolution of Uncertainty Large Risky Personal Savings, Medium Relative Risk Aversion

\begin{tabular}{|c|c|c|c|c|c|}
\hline state & Stocks $t=0$ & Stocks $t=1$ & Stocks $t=2$ & Stocks $t=3$ & Stocks $t=4$ \\
\hline 0,0 & 0.82 & 0.86 & 0.91 & 0.98 & 1.05 \\
0,1 & & 0.72 & 0.76 & 0.80 & 0.85 \\
0,2 & & & 0.65 & 0.67 & 0.71 \\
0,3 & & & & 0.59 & 0.61 \\
0,4 & & & & & 0.54 \\
\hline 1,0 & & 0.99 & 1.05 & 1.13 & 1.20 \\
1,1 & 0.81 & 0.85 & 0.91 & 0.97 \\
1,2 & & & 0.71 & 0.75 & 0.79 \\
1,3 & & & & 0.64 & 0.66 \\
1,4 & & & 1.33 & 1.39 & 0.58 \\
\hline 2,0 & & 0.99 & 1.06 & 1.45 \\
2,1 & & & & 0.85 & 0.90 \\
2,2 & & & & 0.70 & 0.74 \\
2,3 & & & & 1.99 & 0.62 \\
2,4 & & & & 1.32 & 1.96 \\
\hline 3,0 & & & & 0.98 & 1.40 \\
3,1 & & & & & 0.78 \\
3,2 & & & & & 0.83 \\
3,3 & & & & & 3.72 \\
3,4 & & & & & 1.33 \\
\hline 4,0 & & & & & 0.97 \\
4,1 & & & & & \\
4,2 & & & & & \\
4,3 & & & & & \\
4,4 & & & & & \\
\hline
\end{tabular}

1. The market return is i.i.d. with expected value of $11 \%$ and volatility of $20 \%$. The one-period risk-free rate is (1/0.95)-1 and non-stochastic.

2. The date 0 -expected value of the personal savings is $E_{0}\left(S_{T}\right)=200$ with volatility $\sigma_{L}=0.25$. Initial investible wealth is $W_{0}=100$.

3. The coefficient of relative risk aversion is $r=3$.

4. Column 1 shows the state $i, j$, where $i$ is the number of down-moves in the market return process and $j$ is the number of down-moves in the personal savings process.

5. Columns 2-6 show the proportion of wealth invested in stocks in the various states. 
Table 6: Stock Proportions: Equal Resolution of Uncertainty Large Risky Personal Savings, High Relative Risk Aversion

\begin{tabular}{|c|c|c|c|c|c|}
\hline state & Stocks $t=0$ & Stocks $t=1$ & Stocks $t=2$ & Stocks $t=3$ & Stocks $t=4$ \\
\hline 0,0 & 0.45 & 0.49 & 0.55 & 0.62 & 0.73 \\
\hline 0,1 & & 0.42 & 0.45 & 0.50 & 0.57 \\
\hline 0,2 & & & 0.39 & 0.42 & 0.46 \\
\hline 0,3 & & & & 0.36 & 0.39 \\
\hline 0,4 & & & & & 0.34 \\
\hline 1,0 & & 0.52 & 0.59 & 0.68 & 0.81 \\
\hline 1,1 & & 0.44 & 0.48 & 0.54 & 0.62 \\
\hline 1,2 & & & 0.41 & 0.44 & 0.49 \\
\hline 1,3 & & & & 0.38 & 0.41 \\
\hline 1,4 & & & & & 0.35 \\
\hline 2,0 & & & 0.66 & 0.77 & 0.90 \\
\hline 2,1 & & & 0.52 & 0.59 & 0.68 \\
\hline 2,2 & & & 0.43 & 0.47 & 0.53 \\
\hline 2,3 & & & & 0.40 & 0.43 \\
\hline 2,4 & & & & & 0.37 \\
\hline 3,0 & & & & 0.91 & 1.07 \\
\hline 3,1 & & & & 0.66 & 0.77 \\
\hline 3,2 & & & & 0.51 & 0.58 \\
\hline 3,3 & & & & 0.42 & 0.46 \\
\hline 3,4 & & & & & 0.39 \\
\hline 4,0 & & & & & 1.41 \\
\hline 4,1 & & & & & 0.92 \\
\hline 4,2 & & & & & 0.65 \\
\hline 4,3 & & & & & 0.50 \\
\hline 4,4 & & & & & 0.41 \\
\hline
\end{tabular}

1. The market return is i.i.d. with expected value of $11 \%$ and volatility of $20 \%$. The one-period risk-free rate is (1/0.95)-1 and non-stochastic.

2. The date 0 -expected value of the personal savings is $E_{0}\left(S_{T}\right)=200$ with volatility $\sigma_{L}=0.25$. Initial investible wealth is $W_{0}=100$.

3. The coefficient of relative risk aversion is $r=5$.

4. Column 1 shows the state $i, j$, where $i$ is the number of down-moves in the market return process and $j$ is the number of down-moves in the personal savings process.

5. Columns 2-6 show the proportion of wealth invested in stocks in the various states. 


\section{Table 7: Uncertainty Resolution and Stock Proportions}

\begin{tabular}{|c|c|ccc|}
\hline Period 1 & $\alpha$ & 0.25 & 0.5 & 0.75 \\
\hline & $r=2$ & 1.35 & 1.30 & 1.36 \\
& $r=5$ & 0.39 & 0.40 & 0.46 \\
& $r=10$ & 0.14 & 0.16 & 0.19 \\
& $r=20$ & 0.04 & 0.06 & 0.08 \\
\hline \multirow{3}{*}{ Period 2 } & $\alpha$ & 0.25 & 0.50 & 0.75 \\
\hline & $r=2$ & 1.46 & 1.55 & 1.75 \\
& $r=5$ & 0.49 & 0.51 & 0.59 \\
& $r=10$ & 0.20 & 0.23 & 0.25 \\
& $r=20$ & 0.075 & 0.085 & 0.10 \\
\hline
\end{tabular}

1. The market return is i.i.d. with expected value of $11 \%$ and volatility of $20 \%$. The one-period risk-free rate is (1/0.95)-1 and non-stochastic.

2. The date 0-expected value of the personal savings is $E_{0}\left(S_{T}\right)=214$. Initial investible wealth is $W_{0}=100$.

3. The coefficient of relative risk aversion $r$ is assumed to be $2,5,10$, or 20 . In the zero personal-savings case, this leads to constant optimal-stock proportions of $64 \%, 22 \%, 8 \%$, $3 \%$ respectively.

4. The proportion $\alpha=0.25,0.5,0.75$ of the uncertainty of personal savings is resolved over the first period.

5. In year 2, the stock proportions vary over the different states of the market return and the personal-savings expectation. In the bottom half of the table we report the simple average of the stock proportions over the various states. 


\section{Table 8: Wealth Allocations: With and Without Savings Uncertainty}

\begin{tabular}{|c|c|ccccc|}
\hline & state at $t=1$ & 0 & 1 & 2 & 3 & 4 \\
\hline$\sigma_{s}=0$ & & & & & & \\
\hline & $W_{1}$ & 170 & 140 & 111 & 85 & 62 \\
\hline & state at $t=2$ & \multicolumn{5}{|c|}{$W_{2} \mid W_{1}$} \\
\hline & 0 & 244 & 201 & 164 & 131 & 102 \\
& 1 & 223 & 183 & 147 & 117 & 90 \\
& 2 & 195 & 159 & 126 & 98 & 74 \\
& 3 & 160 & 127 & 99 & 74 & 52 \\
& 4 & 113 & 86 & 63 & 43 & 25 \\
\hline & & & & & & \\
\hline$\sigma_{s}=0.50$ & & & & & & \\
\hline & $W_{1}$ & 158 & 133 & 110 & 89 & 71 \\
\hline & state at $t=2$ & & 5 & $W_{2} \mid W_{1}$ & & \\
\hline & 0 & 220 & 184 & 152 & 125 & 101 \\
& 1 & 204 & 169 & 140 & 114 & 92 \\
& 2 & 182 & 150 & 123 & 100 & 79 \\
& 3 & 153 & 126 & 102 & 81 & 63 \\
& 4 & 116 & 93 & 74 & 57 & 42 \\
\hline
\end{tabular}

1. The market return is i.i.d. with expected value of $11 \%$ and volatility of $20 \%$. The one-period risk-free rate is (1/0.95)-1 and non-stochastic.

2. The date 0 -expected value of the personal savings is $E_{0}\left(S_{T}\right)=100$ with volatility $\sigma_{S}=0$, or $\sigma_{S}=0.50$. Initial investible wealth is $W_{0}=100$.

3. The coefficient of relative risk aversion is $r=2$.

4. Wealth allocations chosen are for $t=1$ and $t=2$, for binomial density $n=4$. There are five states of the market portfolio at time $t=1$, indexed by the number of down moves of the binomial process, $i=0,1,2,3,4$. Similarly, there are five states of the market portfolio at $t=2$ conditional on each state at $t=1$.

5. The $t=2$ allocations are those given the central date 1 -state for the personal savings, i.e. the state where expectations of personal savings are unchanged. 
Table 9a: Stock Proportions: Restricted Borrowing ( $\leq 100 \%)$ Large, Positive, Risky Personal Savings

\begin{tabular}{|c|c|c|c|c|c|}
\hline state & Stocks $t=0$ & Stocks $t=1$ & Stocks $t=2$ & Stocks $t=3$ & Stocks $t=4$ \\
\hline 0,0 & 1.29 & 1.30 & 1.32 & 1.34 & 1.35 \\
0,1 & & 1.09 & 1.11 & 1.12 & 1.14 \\
0,2 & & & 0.95 & 0.96 & 0.98 \\
0,3 & & & & 0.85 & 0.86 \\
0,4 & & & & & 0.78 \\
\hline 1,0 & & 1.61 & 1.61 & 1.62 & 1.61 \\
1,1 & & 1.31 & 1.33 & 1.34 & 1.35 \\
1,2 & & & 1.10 & 1.11 & 1.13 \\
1,3 & & & & 0.94 & 0.96 \\
1,4 & & & 2.00 & 2.00 & 0.95 \\
\hline 2,0 & & & 1.68 & 1.68 & 1.34 \\
2,1 & & & & 1.35 & 1.36 \\
2,2 & & & & & 1.12 \\
2,3 & & & & 2.00 & 0.94 \\
2,4 & & & & 1.77 & 2.00 \\
\hline 3,0 & & & & 1.38 & 1.75 \\
3,1 & & & & & 1.38 \\
3,2 & & & & & 2.00 \\
3,3 & & & & & 2.00 \\
3,4 & & & & & 1.88 \\
\hline 4,0 & & & & & \\
4,1 & & & & & \\
4,2 & & & & & \\
4,3 & & & & & \\
4,4 & & & & & \\
\hline
\end{tabular}

1. The market return is i.i.d. with expected value of $11 \%$ and volatility of $20 \%$. The one-period risk-free rate is (1/0.95)-1 and non-stochastic.

2. The date 0-expected value of the personal savings is $E_{0}\left(S_{T}\right)=200$ with volatility $\sigma_{S}=0.25$. Initial investible wealth is $W_{0}=100$. Borrowing is restricted to tradable wealth.

3. The coefficient of relative risk aversion is $r=2$.

4. Column 1 shows the state $i, j$, where $i$ is the number of down-moves in the market return process and $j$ is the number of down-moves in the personal savings process.

5. Columns 2-6 show the proportion of wealth invested in stocks in the various states. 
Table 9b: Stock Proportions: Restricted Borrowing ( $\leq \mathbf{5 0 \%})$ Large, Positive, Risky Personal Savings

\begin{tabular}{|c|c|c|c|c|c|}
\hline state & Stocks $t=0$ & Stocks $t=1$ & Stocks $t=2$ & Stocks $t=3$ & Stocks $t=4$ \\
\hline 0,0 & $\mathbf{1 . 2 8}$ & 1.29 & 1.31 & 1.33 & 1.35 \\
0,1 & & 1.09 & 1.11 & 1.12 & 1.14 \\
0,2 & & & 0.95 & 0.96 & 0.98 \\
0,3 & & & & 0.85 & 0.96 \\
0,4 & & & & & 0.78 \\
\hline 1,0 & & 1.50 & 1.50 & 1.50 & 1.50 \\
1,1 & & 1.30 & $\mathbf{1 . 3 2}$ & 1.34 & 1.35 \\
1,2 & & & 1.09 & 1.11 & 1.13 \\
1,3 & & & & 1.05 & 0.96 \\
1,4 & & & 1.50 & 1.50 & 0.85 \\
\hline 2,0 & & & 1.50 & 1.50 & 1.50 \\
2,1 & & & & 1.34 & 1.30 \\
2,2 & & & & 1.10 & 1.12 \\
2,3 & & & & 1.50 & 1.06 \\
2,4 & & & & 1.50 & 1.50 \\
\hline 3,0 & & & & 1.36 & 1.50 \\
3,1 & & & & & 1.38 \\
3,2 & & & & & 1.50 \\
3,3 & & & & & 1.50 \\
3,4 & & & & & 1.50 \\
\hline 4,0 & & & & & \\
4,1 & & & & & \\
4,2 & & & & & \\
4,3 & & & & & \\
4,4 & & & & & \\
\hline
\end{tabular}

1. The market return is i.i.d. with expected value of $11 \%$ and volatility of $20 \%$. The one-period risk-free rate is (1/0.95)-1 and non-stochastic.

2. The date 0-expected value of the personal savings is $E_{0}\left(S_{T}\right)=200$ with volatility $\sigma_{S}=0.25$. Initial investible wealth is $W_{0}=100$. Borrowing is restricted to $50 \%$ of tradable wealth.

3. The coefficient of relative risk aversion is $r=2$.

4. Column 1 shows the state $i, j$, where $i$ is the number of down-moves in the market return process and $j$ is the number of down-moves in the personal savings process.

5. Columns 2-6 show the proportion of wealth invested in stocks in the various states. 
Table 9c: Stock Proportions: Restricted Borrowing ( $\leq$ 0\%) Large, Positive, Risky Personal Savings

\begin{tabular}{|c|c|c|c|c|c|}
\hline state & Stocks $t=0$ & Stocks $t=1$ & Stocks $t=2$ & Stocks $t=3$ & Stocks $t=4$ \\
\hline 0,0 & $\mathbf{1 . 0 0}$ & 1.00 & 1.00 & 1.00 & 1.00 \\
0,1 & & 1.00 & 1.00 & 1.00 & 1.00 \\
0,2 & & & 0.96 & 0.98 & 1.00 \\
0,3 & & & & 0.86 & 0.88 \\
0,4 & & & & & 0.79 \\
\hline 1,0 & & 1.00 & 1.00 & 1.00 & 1.00 \\
1,1 & & 1.00 & $\mathbf{1 . 0 0}$ & 1.00 & 1.00 \\
1,2 & & & 1.00 & 1.00 & 1.00 \\
1,3 & & & & 0.95 & 0.97 \\
1,4 & & & 1.00 & 1.00 & 0.85 \\
\hline 2,0 & & & 1.00 & 1.00 & 1.00 \\
2,1 & & & & 1.00 & 1.00 \\
2,2 & & & & 1.00 & 1.00 \\
2,3 & & & & 1.00 & 0.94 \\
2,4 & & & & 1.00 & 1.00 \\
\hline 3,0 & & & & 1.00 & 1.00 \\
3,1 & & & & & 1.00 \\
3,2 & & & & & 1.00 \\
3,3 & & & & & 1.00 \\
3,4 & & & & & 1.00 \\
\hline 4,0 & & & & & \\
4,1 & & & & & \\
4,2 & & & & & \\
4,3 & & & & & \\
4,4 & & & & & \\
\hline
\end{tabular}

1. The market return is i.i.d. with expected value of $11 \%$ and volatility of $20 \%$. The one-period risk-free rate is (1/0.95)-1 and non-stochastic.

2. The date 0 -expected value of the personal savings is $E_{0}\left(S_{T}\right)=200$ with volatility $\sigma_{L}=0.25$. Initial investible wealth is $W_{0}=100$. Borrowing is not allowed.

3. The coefficient of relative risk aversion is $r=2$.

4. Column 1 shows the state $i, j$, where $i$ is the number of down-moves in the market return process and $j$ is the number of down-moves in the personal savings process.

5. Columns 2-6 show the proportion of wealth invested in stocks in the various states. 
Long-Term Portfolio Choice Given Uncertain Personal Savings

Table 10: Stock Proportions: Unrestricted Borrowing Medium, Positive, Risky Personal Savings: Part A (assumptions as in Table 2)

\begin{tabular}{|c|c|c|c|c|c|c|c|c|c|c|}
\hline state & $t=0$ & $t=1$ & $t=2$ & $t=3$ & $t=4$ & $t=5$ & $t=6$ & $t=7$ & $t=8$ & $t=9$ \\
\hline 0,0 & 0.89 & 0.90 & 0.91 & 0.92 & 0.94 & 0.95 & 0.96 & 0.98 & 1.01 & 1.03 \\
\hline 0,1 & & 0.82 & 0.83 & 0.84 & 0.85 & 0.86 & 0.88 & 0.89 & 0.91 & 0.92 \\
\hline 0,2 & & & 0.78 & 0.78 & 0.79 & 0.80 & 0.81 & 0.82 & 0.83 & 0.84 \\
\hline 0,3 & & & & 0.75 & 0.75 & 0.76 & 0.76 & 0.76 & 0.77 & 0.78 \\
\hline 0,4 & & & & & 0.73 & 0.73 & 0.73 & 0.73 & 0.73 & 0.73 \\
\hline 0,5 & & & & & & 0.71 & 0.71 & 0.71 & 0.70 & 0.71 \\
\hline 0,6 & & & & & & & 0.70 & 0.69 & 0.69 & 0.69 \\
\hline 0,7 & & & & & & & & 0.69 & 0.68 & 0.68 \\
\hline 0,8 & & & & & & & & & 0.67 & 0.67 \\
\hline 0,9 & & & & & & & & & & 0.67 \\
\hline 1,0 & & 0.98 & 0.99 & 1.00 & 1.01 & 1.02 & 1.04 & 1.05 & 1.10 & 1.12 \\
\hline 1,1 & & 0.87 & 0.89 & 0.90 & 0.91 & 0.92 & 0.94 & 0.95 & 0.98 & 0.99 \\
\hline 1,2 & & & 0.81 & 0.82 & 0.83 & 0.84 & 0.85 & 0.86 & 0.88 & 0.89 \\
\hline 1,3 & & & & 0.77 & 0.78 & 0.78 & 0.79 & 0.80 & 0.81 & 0.82 \\
\hline 1,4 & & & & & 0.74 & 0.74 & 0.75 & 0.75 & 0.75 & 0.76 \\
\hline 1,5 & & & & & & 0.72 & 0.72 & 0.72 & 0.72 & 0.72 \\
\hline 1,6 & & & & & & & 0.71 & 0.70 & 0.70 & 0.70 \\
\hline 1,7 & & & & & & & & 0.69 & 0.68 & 0.69 \\
\hline 1,8 & & & & & & & & & 0.68 & 0.68 \\
\hline 1,9 & & & & & & & & & & 0.67 \\
\hline 2,0 & & & 1.12 & 1.13 & 1.13 & 1.14 & 1.15 & 1.16 & 1.21 & 1.22 \\
\hline 2,1 & & & 0.96 & 0.97 & 0.98 & 1.00 & 1.01 & 1.03 & 1.06 & 1.08 \\
\hline 2,2 & & & 0.86 & 0.87 & 0.89 & 0.90 & 0.91 & 0.92 & 0.95 & 0.96 \\
\hline 2,3 & & & & 0.80 & 0.81 & 0.82 & 0.83 & 0.84 & 0.85 & 0.87 \\
\hline 2,4 & & & & & 0.76 & 0.77 & 0.77 & 0.78 & 0.79 & 0.79 \\
\hline 2,5 & & & & & & 0.73 & 0.74 & 0.74 & 0.74 & 0.75 \\
\hline 2,6 & & & & & & & 0.71 & 0.71 & 0.71 & 0.71 \\
\hline 2,7 & & & & & & & & 0.70 & 0.69 & 0.69 \\
\hline 2,8 & & & & & & & & & 0.68 & 0.68 \\
\hline 2,9 & & & & & & & & & & 0.67 \\
\hline 3,0 & & & & 1.36 & 1.34 & 1.33 & 1.32 & 1.32 & 1.35 & 1.36 \\
\hline 3,1 & & & & 1.10 & 1.11 & 1.11 & 1.12 & 1.14 & 1.17 & 1.19 \\
\hline 3,2 & & & & 0.94 & 0.96 & 0.97 & 0.98 & 1.00 & 1.03 & 1.04 \\
\hline 3,3 & & & & 0.85 & 0.86 & 0.87 & 0.89 & 0.90 & 0.91 & 0.93 \\
\hline 3,4 & & & & & 0.79 & 0.80 & 0.81 & 0.82 & 0.83 & 0.84 \\
\hline 3,5 & & & & & & 0.75 & 0.76 & 0.76 & 0.77 & 0.78 \\
\hline 3,6 & & & & & & & 0.73 & 0.73 & 0.73 & 0.73 \\
\hline 3,7 & & & & & & & & 0.71 & 0.70 & 0.71 \\
\hline 3,8 & & & & & & & & & 0.69 & 0.69 \\
\hline 3,9 & & & & & & & & & & 0.68 \\
\hline 4,0 & & & & & 1.76 & 1.68 & 1.62 & 1.56 & 1.57 & 1.55 \\
\hline 4,1 & & & & & 1.32 & 1.31 & 1.31 & 1.30 & 1.33 & 1.34 \\
\hline 4,2 & & & & & 1.07 & 1.08 & 1.09 & 1.11 & 1.14 & 1.15 \\
\hline 4,3 & & & & & 0.93 & 0.94 & 0.95 & 0.97 & 0.99 & 1.01 \\
\hline 4,4 & & & & & 0.84 & 0.85 & 0.86 & 0.87 & 0.88 & 0.90 \\
\hline 4,5 & & & & & & 0.78 & 0.79 & 0.80 & 0.81 & 0.82 \\
\hline 4,6 & & & & & & & 0.75 & 0.75 & 0.75 & 0.76 \\
\hline 4,7 & & & & & & & & 0.72 & 0.72 & 0.72 \\
\hline 4,8 & & & & & & & & & 0.70 & 0.70 \\
\hline 4,9 & & & & & & & & & & 0.68 \\
\hline
\end{tabular}


Table 10: Stock Proportions: Unrestricted Borrowing Medium, Positive, Risky Personal Savings: Part B (assumptions as in Table 2)

\begin{tabular}{|c|c|c|c|c|c|c|c|c|c|c|}
\hline state & $t=0$ & $t=1$ & $t=2$ & $t=3$ & $t=4$ & $t=5$ & $t=6$ & $t=7$ & $t=8$ & $t=9$ \\
\hline 5,0 & & & & & & 2.48 & 2.25 & 2.06 & $\begin{array}{l}1.93 \\
\end{array}$ & 1.84 \\
\hline 5,1 & & & & & & 1.71 & 1.65 & 1.59 & 1.56 & 1.55 \\
\hline 5,2 & & & & & & 1.29 & 1.28 & 1.28 & 1.29 & 1.31 \\
\hline 5,3 & & & & & & 1.05 & 1.06 & 1.07 & 1.10 & 1.11 \\
\hline 5,4 & & & & & & 0.91 & 0.92 & 0.94 & 0.95 & 0.97 \\
\hline 5,5 & & & & & & 0.82 & 0.83 & 0.84 & 0.86 & 0.87 \\
\hline 5,6 & & & & & & & 0.77 & 0.78 & 0.79 & 0.79 \\
\hline 5,7 & & & & & & & & 0.74 & 0.74 & 0.74 \\
\hline 5,8 & & & & & & & & & 0.71 & 0.71 \\
\hline 5,9 & & & & & & & & & & 0.69 \\
\hline 6,0 & & & & & & & 3.71 & 3.33 & 2.83 & 2.45 \\
\hline 6,1 & & & & & & & 2.45 & 2.21 & 2.01 & 1.91 \\
\hline 6,2 & & & & & & & 1.66 & 1.60 & 1.56 & 1.55 \\
\hline 6,3 & & & & & & & 1.25 & 1.25 & 1.26 & 1.27 \\
\hline 6,4 & & & & & & & 1.02 & 1.04 & 1.06 & 1.07 \\
\hline 6,5 & & & & & & & 0.89 & 0.90 & 0.92 & 0.93 \\
\hline 6,6 & & & & & & & 0.81 & 0.82 & 0.83 & 0.84 \\
\hline 6,7 & & & & & & & & 0.76 & 0.77 & 0.77 \\
\hline 6,8 & & & & & & & & & 0.73 & 0.73 \\
\hline 6,9 & & & & & & & & & & 0.70 \\
\hline 7,0 & & & & & & & & 4.59 & 5.06 & 5.80 \\
\hline 7,1 & & & & & & & & 3.93 & 3.32 & 2.71 \\
\hline 7,2 & & & & & & & & 2.38 & 2.12 & 1.99 \\
\hline 7,3 & & & & & & & & 1.59 & 1.55 & 1.54 \\
\hline 7,4 & & & & & & & & 1.21 & 1.22 & 1.23 \\
\hline 7,5 & & & & & & & & 1.00 & 1.01 & 1.03 \\
\hline 7,6 & & & & & & & & 0.87 & 0.89 & 0.90 \\
\hline 7,7 & & & & & & & & 0.80 & 0.80 & 0.81 \\
\hline 7,8 & & & & & & & & & 0.75 & 0.76 \\
\hline 7,9 & & & & & & & & & & 0.72 \\
\hline 8,0 & & & & & & & & & 2.41 & 31.00 \\
\hline 8,1 & & & & & & & & & 4.49 & 9.51 \\
\hline 8,2 & & & & & & & & & 4.23 & 3.12 \\
\hline 8,3 & & & & & & & & & 2.26 & 2.09 \\
\hline 8,4 & & & & & & & & & 1.53 & 1.52 \\
\hline 8,5 & & & & & & & & & 1.17 & 1.19 \\
\hline 8,6 & & & & & & & & & 0.97 & 0.99 \\
\hline 8,7 & & & & & & & & & 0.85 & 0.87 \\
\hline 8,8 & & & & & & & & & 0.78 & 0.79 \\
\hline 8,9 & & & & & & & & & & 0.74 \\
\hline 9,0 & & & & & & & & & & 31.00 \\
\hline 9,1 & & & & & & & & & & 31.00 \\
\hline 9,2 & & & & & & & & & & 15.23 \\
\hline 9,3 & & & & & & & & & & 4.06 \\
\hline 9,4 & & & & & & & & & & 2.19 \\
\hline 9,5 & & & & & & & & & & 1.48 \\
\hline 9,6 & & & & & & & & & & 1.14 \\
\hline 9,7 & & & & & & & & & & 0.95 \\
\hline 9,8 & & & & & & & & & & 0.83 \\
\hline 9,9 & & & & & & & & & & 0.77 \\
\hline
\end{tabular}




\section{Figure 1}

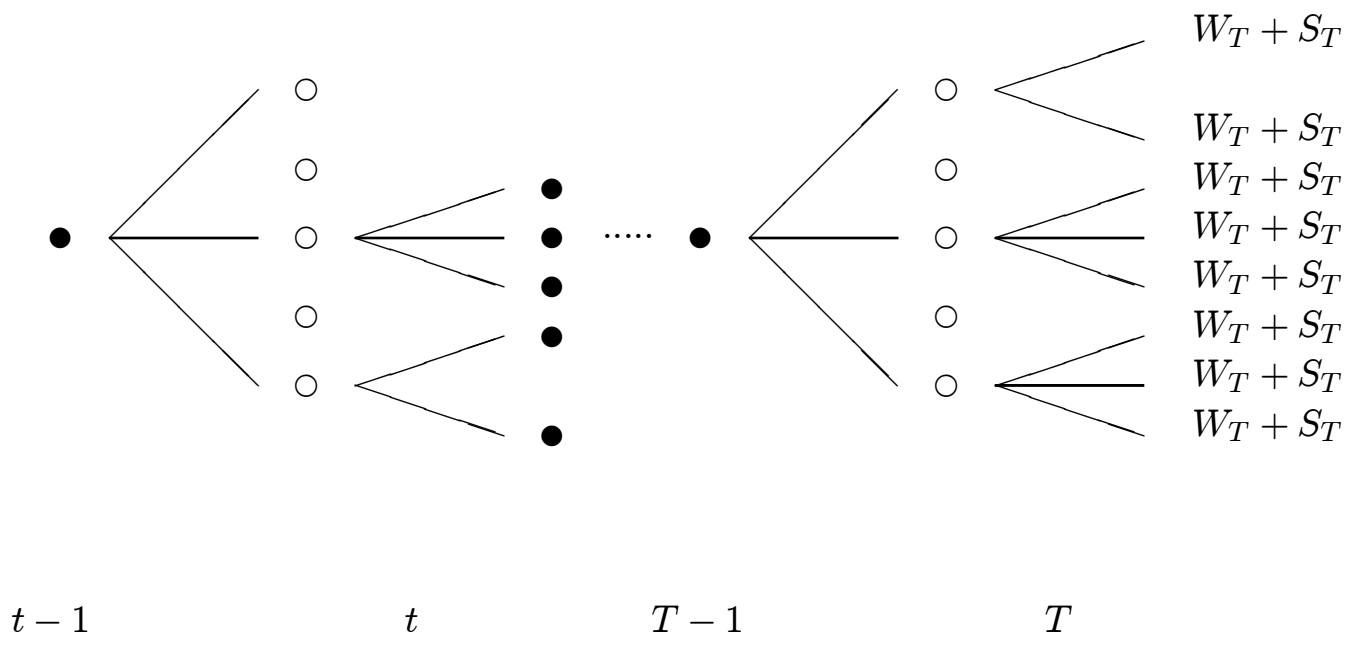

\section{The Tree of Traded and Personal-Savings States}

In Figure 1, the black nodes represent different states of the personal-savings process. The traded states, i.e. the states of the market return, are shown by the clear nodes. At each date, the investor buys claims contingent on a traded state at the following date. In general, the two stochastic processes may be correlated, and the market return process can be mean reverting. 


\section{Figure 2a: Optimal Portfolio Strategy Large, Risky Personal Savings}

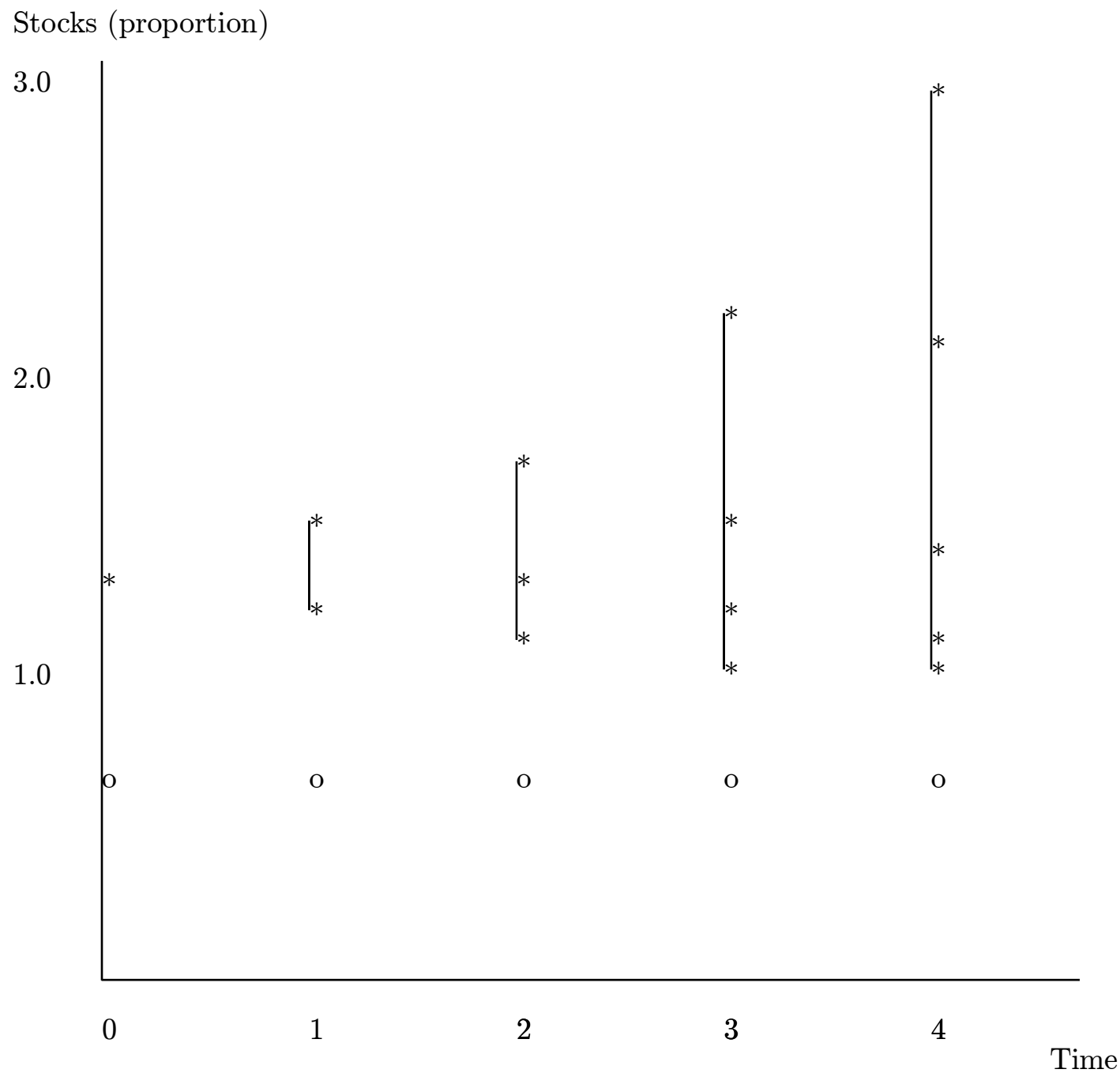

Notes:

1. The data are the same as in Table $1, E_{0}\left(S_{T}\right)=200$. 


\section{Figure 2b: Optimal Portfolio Strategy} Medium Size, Risky Personal Savings

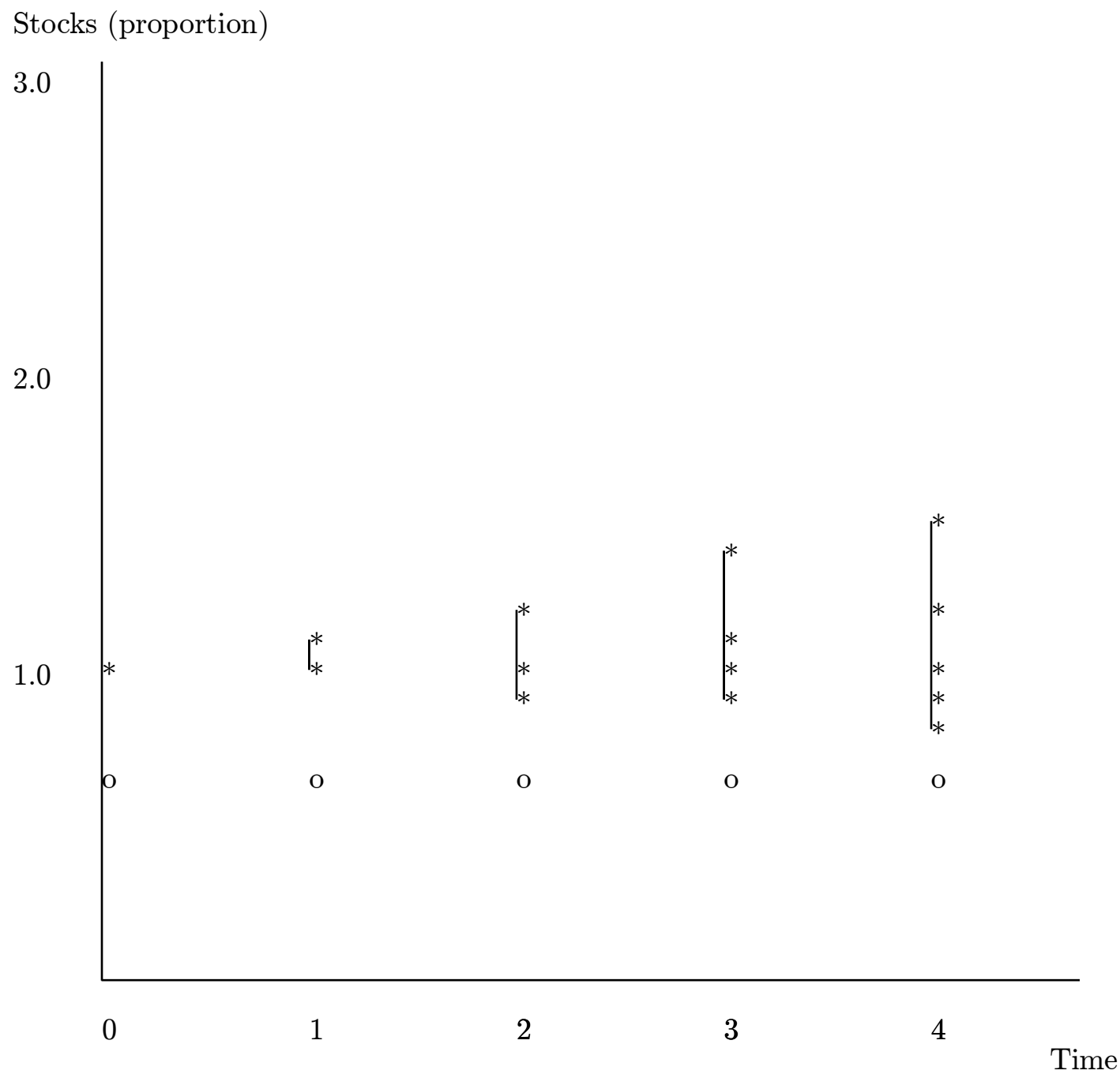

Notes:

1. The data are the same as in Table $2, E_{0}\left(S_{T}\right)=100$. 


\section{Figure 2c: Optimal Portfolio Strategy No Personal Savings}

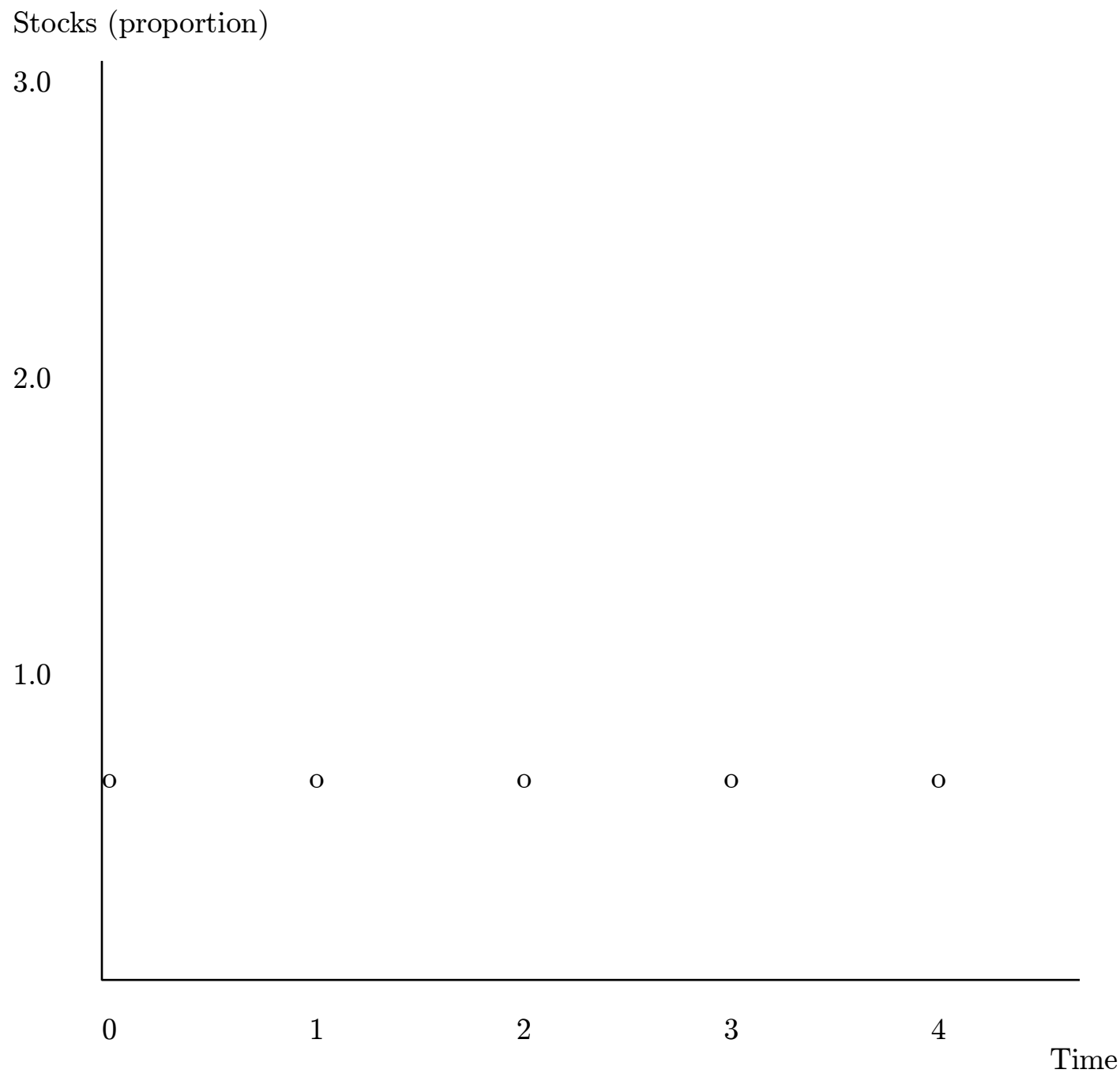

Notes:

1. The data are the same as in Table 1 , except that $E_{0}\left(S_{T}\right)=0$. 


\section{Figure 3a: Optimal Portfolio Strategy Medium Size, Risky Personal Savings}

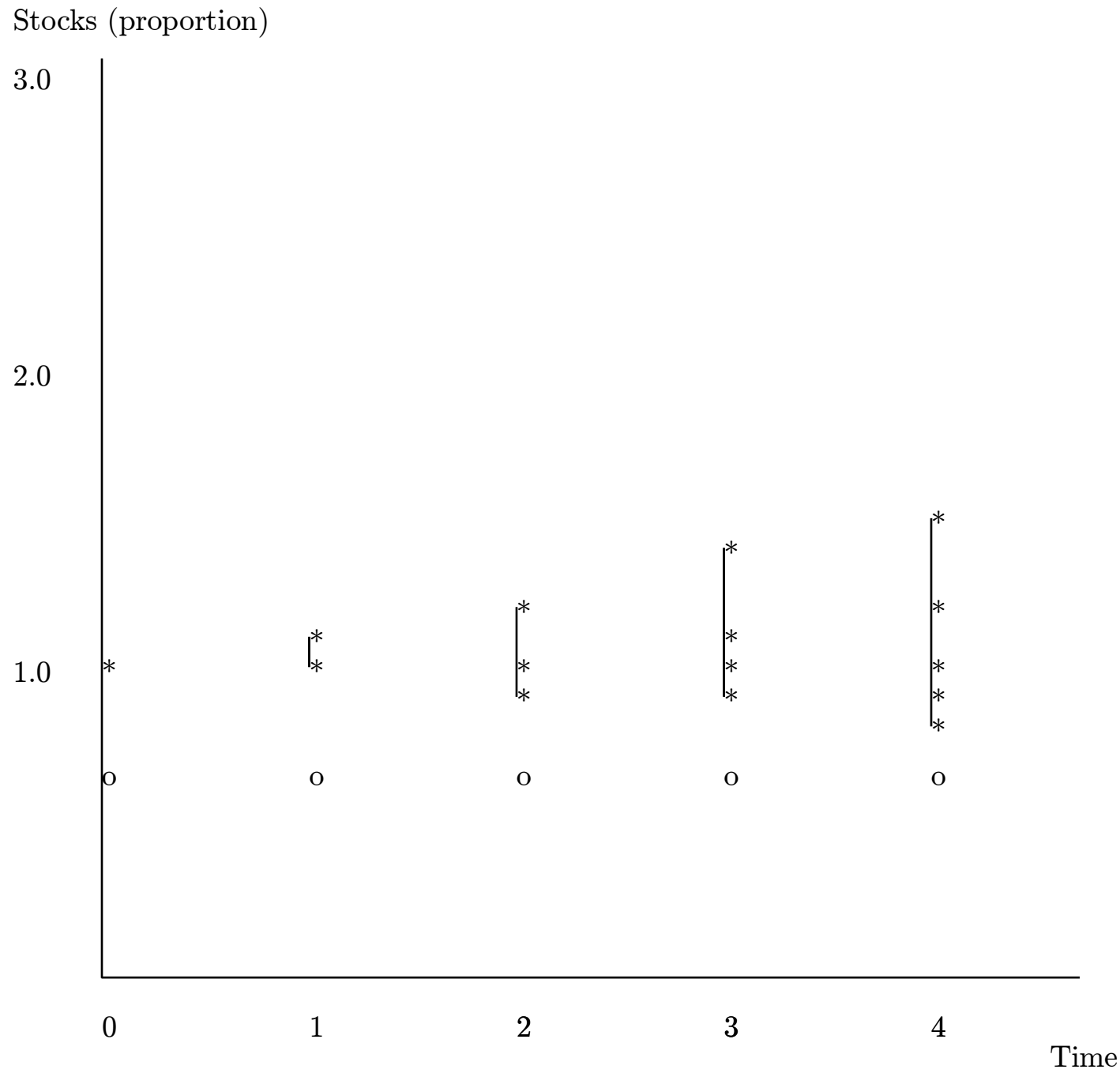

Notes:

1. The data are the same as in Table 2. 
Figure 3b: Optimal Portfolio Strategy Medium Size, Risk-free Personal Savings

Stocks (proportion)

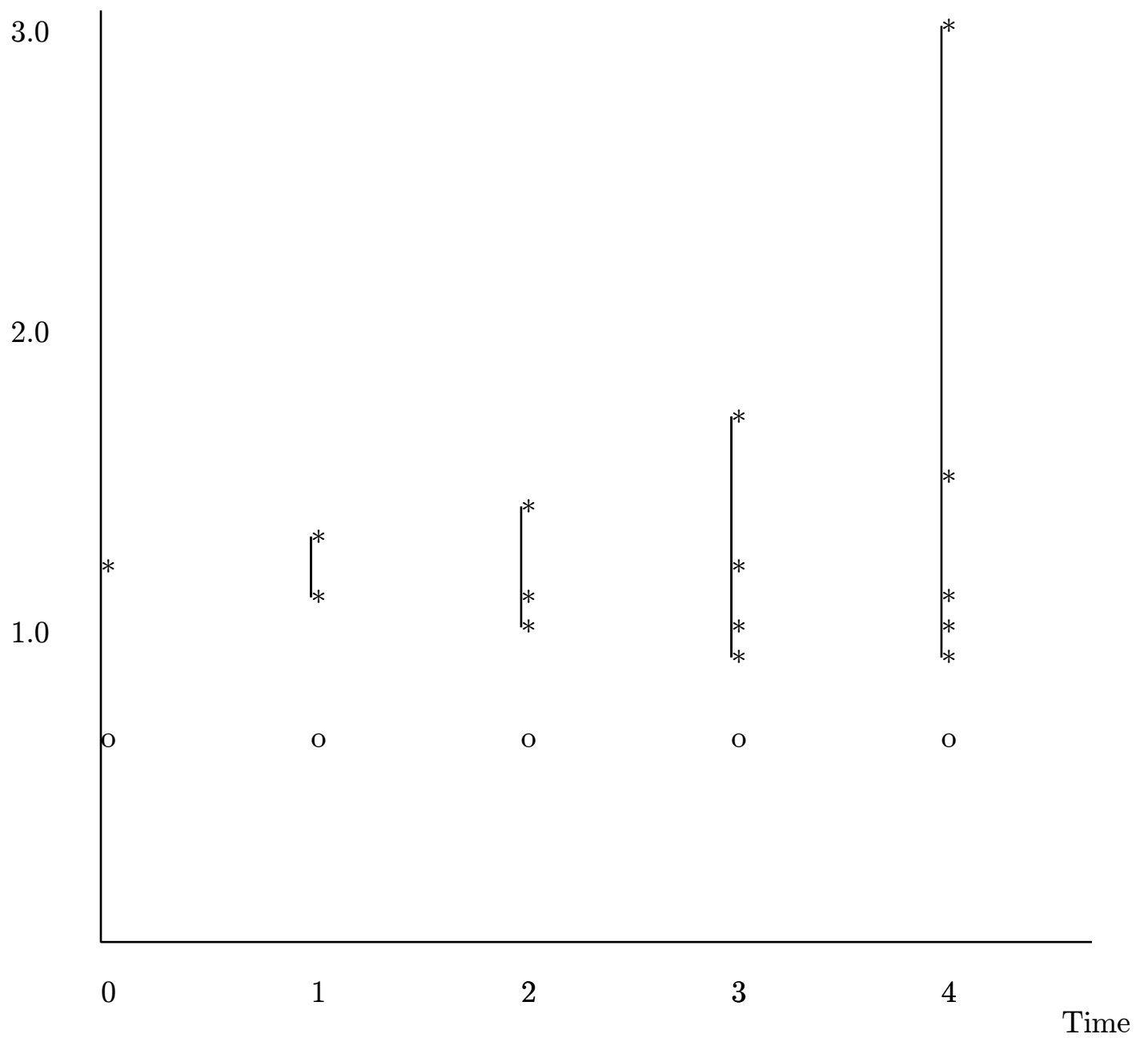

Notes:

1. The data are the same as in Table 3 . 
Figure 3c: Optimal Portfolio Strategy Medium Size, Correlated Risky Personal Savings

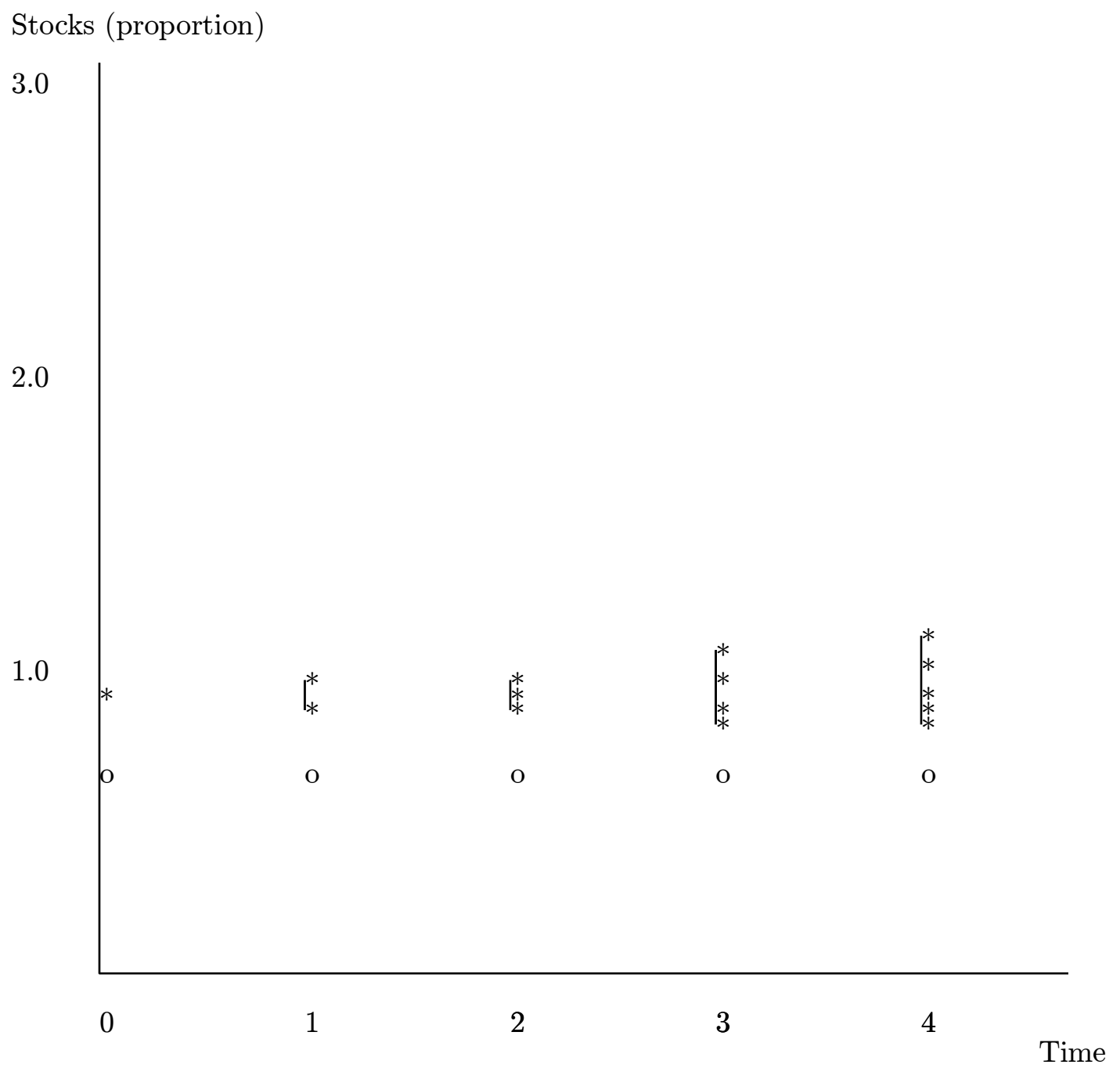

Notes:

1. The data are the same as in Table 4. 
Figure 4a: Optimal Portfolio Strategy Low Relative Risk Aversion, Large Risky Personal Savings

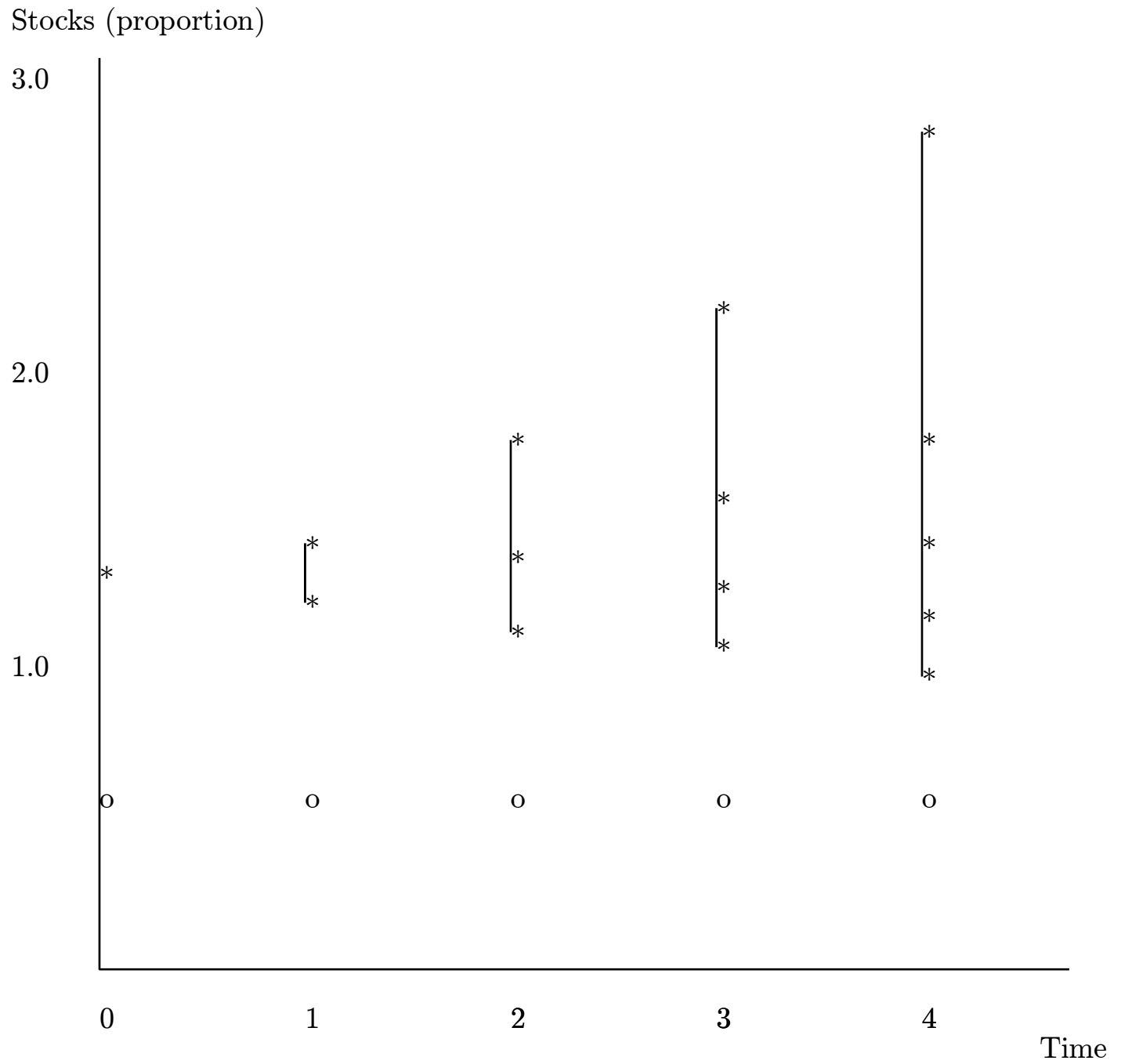

Notes:

1. The data are the same as in Table $1, r=2$. 
Figure 4b: Optimal Portfolio Strategy Medium Relative Risk Aversion, Large Risky Personal Savings

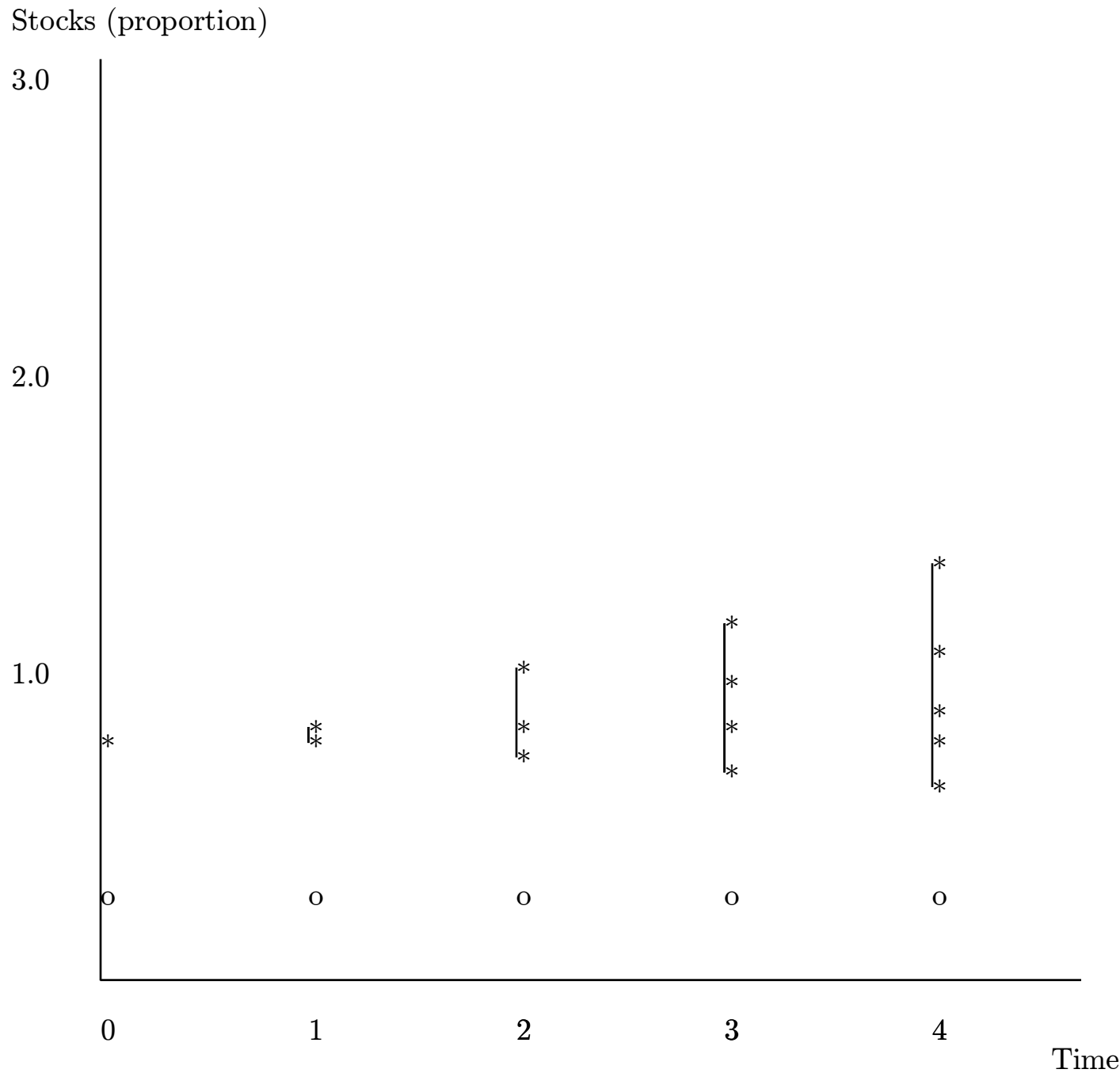

Notes:

1. The data are the same as in Table $5, r=3$. 
Figure 4c: Optimal Portfolio Strategy High Relative Risk Aversion, Large Risky Personal Savings

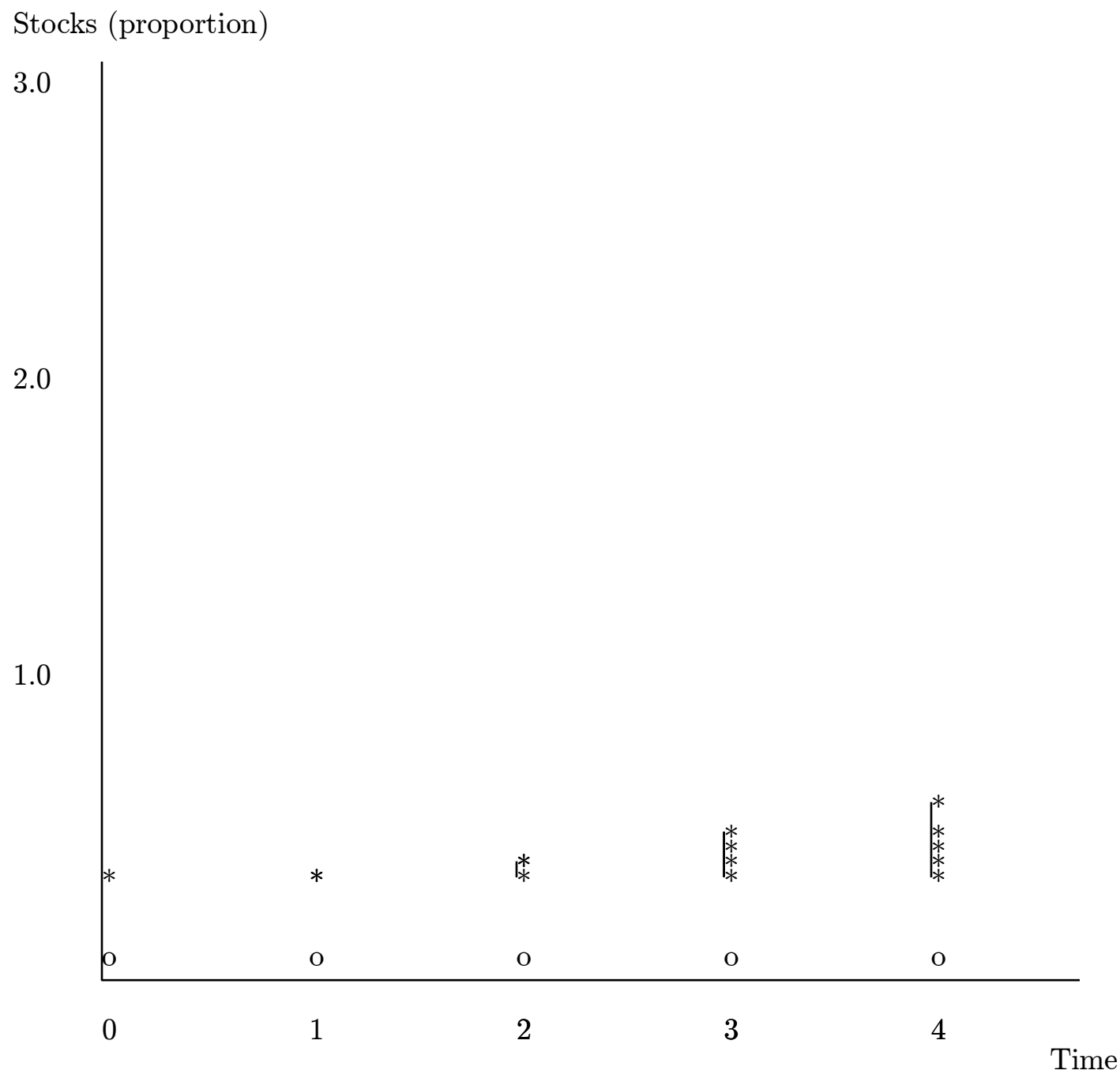

Notes:

1. The data are the same as in Table $6, r=5$. 